\title{
A Systematic Review of Applications of Machine Learning Techniques for Wildfire Management Decision Support
}

\author{
Karol Bot* and José G. Borges (D)
}

Forest Research Center and Laboratory Terra, School of Agriculture, University of Lisbon, Tapada da Ajuda, 1349-017 Lisboa, Portugal; joseborges@isa.ulisboa.pt

* Correspondence: karolbot@isa.ulisboa.pt

check for

updates

Citation: Bot, K.; Borges, J.G. A Systematic Review of Applications of Machine Learning Techniques for Wildfire Management Decision Support. Inventions 2022, 7, 15. https://doi.org/10.3390/ inventions7010015

Academic Editor: Anastasios Doulamis

Received: 3 December 2021 Accepted: 17 January 2022 Published: 21 January 2022

Publisher's Note: MDPI stays neutral with regard to jurisdictional claims in published maps and institutional affiliations.

Copyright: (C) 2022 by the authors. Licensee MDPI, Basel, Switzerland. This article is an open access article distributed under the terms and conditions of the Creative Commons Attribution (CC BY) license (https:// creativecommons.org/licenses/by/ $4.0 /)$.

\begin{abstract}
Wildfires threaten and kill people, destroy urban and rural property, degrade air quality, ravage forest ecosystems, and contribute to global warming. Wildfire management decision support models are thus important for avoiding or mitigating the effects of these events. In this context, this paper aims at providing a review of recent applications of machine learning methods for wildfire management decision support. The emphasis is on providing a summary of these applications with a classification according to the case study type, machine learning method, case study location, and performance metrics. The review considers documents published in the last four years, using a sample of 135 documents (review articles and research articles). It is concluded that the adoption of machine learning methods may contribute to enhancing support in different fire management phases.
\end{abstract}

Keywords: wildfires; machine learning; applications; decision support; review

\section{Introduction}

Wildfires, e.g., uncontrolled fires occurring in forest or grassland in rural areas [1], threaten and kill people, destroy urban and rural property, degrade air quality, ravage forest ecosystems and Natura 2000 sites, and contribute to global warming. The connection between climate change and the increased risk of wildfires suggests a paradigm change in the co-existence of humans with natural catastrophes affecting the environment [2]. Indeed, the regime of wildfires in the Anthropocene is changing due to this complex firehuman-climate interaction [3]. The forest fires paradox has been highlighted by several authors [4-6]. They may play an important ecological role by removing deadwood and opening space for the growth of new vegetation. They may also release nutrients into the soil and offer ecological niches for the proliferation of wildlife. In contrast, when occurring at high intensity, forest fires lead to negative environmental impacts such as a decrease in soil quality (e.g., loss of biota, volatilization of its nutrients, and an increase in erosion). They may further contribute to a decline in biodiversity, as well as to a decrease in air quality [3,4], thus threatening forested landscapes [7].

Wildfires result from the interaction of several factors (e.g., the composition of the fuels, ignition sources, weather conditions, and topography) [8]. The landscape mosaic impacts the wildfire development process, e.g., fire ignition and frequency, rate of spread, the energy released, and the severity [3]. The complexity of the phenomenon poses a challenge to its modelling and simulation in order to address wildfire hazards proactively, i.e., to enhance silvicultural practices and forest management plans to design resilient landscapes and to reduce loss [9-12] According to the EU Horizon 2020 Work Programme [11], the fire management cycle may be broadly segmented into three stages: (i) prevention and preparedness (pre-fire); (ii) detection and response (management of active wildfires); (iii) restoration and adaptation activities (post-wildfire). The literature discusses the research into methods and tools to help address each stage, as well as the policy emphasis on each $[12,13]$. 
The literature reports a variety of models targeting specific stages of the fire management cycle, e.g., wildfire occurrence models, wildfire damage models (e.g., [14-18]), wildfire spread models $[9,12,19]$, fuel and stand growth and yield models, stand-level management scheduling methods, forested landscape management methods (e.g., [20,21]), dispatch and deployment models, and information and decision systems as technological support platforms (e.g., [15-17,22]). Scientific methods, namely, statistical modelling and operations research analysis have contributed greatly to understanding the behaviour and driving factors of wildfires, as well as providing situational awareness and decision support to improve operational decision-making [23,24]. Nevertheless, despite scientific and technological breakthroughs in addressing specific fire management problems, new approaches are needed to understand the complex wildfire phenomenon and mitigate its impacts [3]. The use of machine learning models is a well-established approach in many fields of science $[20,21]$, and could thus be an option to address the challenges faced by wildfire management.

According to [25], "machine learning is an evolving branch of computational algorithms that are designed to emulate human intelligence by learning from the surrounding environment". Furthermore, it "can improve automatically through experience" [8]. In [8], the most frequently used machine learning approaches are detailed from a wildfire perspective. The authors briefly present popular algorithms within each category (supervised, unsupervised, and agent-based), along with references that discuss the fundamentals of machine learning methods. Details considering the theory of supervised learning may be found in [26-28]. Supervised learning aims to map labelled input to known output, using a continuous target variable or a categorical target variable. The continuous target variable is used for regression tasks, with possible applications in this context in fire susceptibility, fire spread/burn area prediction, fire occurrence, fire severity, smoke prediction, and climate change assessment [8]. Some of the popular algorithms for regression tasks are: naive Bayes [29], decision trees [30], classification and regression trees [31], random forest [32], deep neural network [33], Gaussian processes [34], neural networks [35], genetic algorithms [36], recurrent neural networks [37], and maximum entropy [38]. The categorical target variable is used for classification tasks, with possible applications in this context in fuel characterization, fire detection and fire mapping, for example [8]. Popular algorithms for classification tasks are: neural networks, decision trees, boosted regression trees (gradient boosted machine) [39], random forest, K-nearest neighbour [40], and support vector machines [41]. Details on the theory of unsupervised learning may be found in [28]. Unsupervised learning aims to understand patterns and discover outputs, using data in which the target variable is not available. It can be used for clustering and dimensionality reduction tasks. Possible applications for the clustering task, in this scope, are fire detection, fire mapping, burned area prediction, and fire weather prediction [8]. Popular algorithms for clustering tasks are: K-means clustering [42], self-organizing maps [43], autoencoders, Gaussian mixture models [44], ISODATA, hidden Markov models [45], and hard competitive learning [46]. Possible applications for the dimensionality reduction task are landscape controls on fire, fire susceptibility, and fire spread/burned area prediction [8]. Popular algorithms for dimensionality reduction tasks are: self-organizing maps, autoencoders, t-distributed stochastic neighbour embedding [47], random forest, boosted regression trees (also known as gradient boosted machine), and maximum entropy.

Nevertheless, as highlighted by the literature, innovations in wildfire management decision support have consisted mostly of advancements in the field of operational research approaches. There is little experience of taking advantage of the potential of machine learning or deep reinforcement learning techniques-as briefly outlined above-to enhance wildfire management decision support (e.g., [3,48]). This provided the motivation for a review of machine learning techniques and applications that may provide insights into their potential to address the complexity of a holistic approach to fire management. This review considers recent (2019-present) applications of machine learning methods for wildfire management decision support, considering the last four years of publications. The 
emphasis is on providing a summary and a classification of the applications according to the study type, main application of the model, machine learning technique, case study location, and performance metrics.

\section{Objective, Contribution, and Organization of the Work}

The present work aims at developing a review of the recent applications of machine learning methods for wildfire management decision support, considering the last four years of publications (from 2019 to the present). This review aims at providing the following contributions:

- A summary of the applications developed by the studies mentioned;

- A classification according to the study type, main application of the model, machine learning technique, case study location, and performance metrics.

The work is organized into four main sections. Section 1 provides an introduction to the scope of the work and its objectives and contributions. Section 2 presents the methodology used for the development of the systematic review. Section 3 presents references to the basics of wildfire modelling and machine learning methods, fundamentally presenting the review results. Section 4 presents the conclusions.

\section{Systematic Review Methodology}

\subsection{Database and Search Terms}

The review was built from the Clarivate Web of Science database. The search terms were ("machine learning" OR "computational learning") AND ("wildfire" OR "fire").

\subsection{Eligibility Criteria}

The criteria for selecting results from the database queries were: (i) a time interval of four years (from 2019 to the present); (ii) the document type being a journal research article or review article; (iii) a pertinent match of the title and abstract to the objective of this work (a focus on wildfire modelling using machine learning techniques); (iv) detailed consideration of the whole document to assess its relevance (description of the machine learning technique used, description of the wildfire modelling application, description of the input data and main results).

\subsection{Data Collection Results}

Figure 1 presents the results (in numeric terms) for each filtering stage in the systematic review methodology. The initial search comprised 682 documents, and the final stage (where the documents were considered eligible and are included in this review) comprised 135 documents.

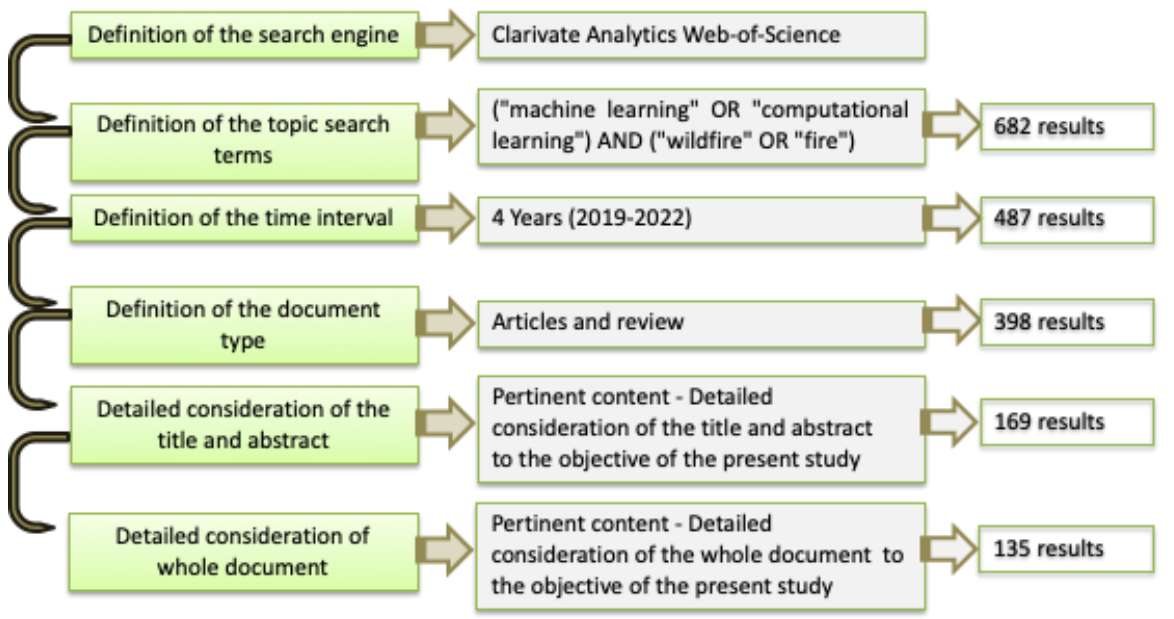

Figure 1. Schematic of search method and obtained results for each document filtering stage. 


\section{Results}

The results highlighted four earlier reviews of applications of machine learning methods to wildfire management. In Section 3.1, we list these reviews and highlight further the motivation for this new review and its added value. Subsequently, we present a summary of all papers with an emphasis on the methods and techniques used (Section 3.2). The methods' input features and feature selection are further explored in Section 3.3. Finally, Section 3.4 discusses the research trends, challenges, and prospects regarding the applications of machine-learning-based methods to wildfire management.

\subsection{Related Reviews}

Three review papers were identified within the final sample of 135 studies focusing on machine learning applications in wildfire management decision support (Table 1). In contrast with these reviews, the present work classifies the documents according to the wildfire management decision support stage, namely, (i) prevention and other related prefire decision support applications, (ii) active wildfire-related decision support applications, and (iii) restoration and other post-fire-related decision support applications. Moreover, this review has a broader scope and updates the findings from earlier reviews, as highlighted in the following sub-sections.

Table 1. Results: pertinent review articles and addressed issues.

\begin{tabular}{ccc}
\hline Ref. & Title & Addressed Issues \\
\hline [8] & $\begin{array}{c}\text { A review of machine learning } \\
\text { applications in wildfire science and } \\
\text { management }\end{array}$ & $\begin{array}{c}\text { Overview of popular machine learning methods, } \\
\text { review of applications and advantages and } \\
\text { limitations of the methods }\end{array}$ \\
\hline [49] & $\begin{array}{c}\text { Forest fire induced Natech risk } \\
\text { assessment: A survey of geospatial } \\
\text { technologies }\end{array}$ & $\begin{array}{c}\text { Review methods based on geospatial } \\
\text { information systems (GIS) for modelling wildfire } \\
\text { risk and their Natural Hazards Triggering } \\
\text { Technological Disasters (Natech) potential }\end{array}$ \\
\hline 50$]$ & $\begin{array}{c}\text { A Survey of Machine Learning } \\
\text { Algorithms Based Forest Fires } \\
\text { Prediction and Detection Systems }\end{array}$ & $\begin{array}{c}\text { Review of various methods used in forest fires } \\
\text { prediction and detection }\end{array}$ \\
\hline
\end{tabular}

\subsection{Machine-Learning-Based Applications}

The applications are classified into three main categories: pre-fire prevention, active wildfire, and restoration and post-fire. We outline the application, machine learning technique employed, case study location, and model performance metrics in Tables 2-4, for the documents in which there is comprehensive description of the machine learning method employed. This outline is intended to support the discussion of results and facilitate further reference in the case of applications where the machine learning method is fully described.

\subsubsection{Pre-Fire Prevention and Preparedness}

The 51 papers addressing this wildfire cycle management phase focused on the following applications: wildfire fuel modelling, risk assessment and ignition prediction of wildfires, support to dispatch, landscape planning and prevention measures for severity mitigation, and development of inventory data. Of the 51 articles, 13.7\% applied machine learning to wildfire fuel modelling (A), 78.4\% to risk assessment and ignition prediction of wildfires (B), nearly $2.0 \%$ to landscape planning and prevention measures for severity mitigation (D), and 5.9\% to the development of inventory data (E).

\section{Wildfire Fuel Modelling}

In [51], The improvement of the monitoring of Fuel Management Zones has been studied using extreme gradient boosting, support vector machine, random forest, and K-nearest neighbours machine learning methods, fed with data from satellite images, vege- 
tation indices and temporal characteristics. In [52], the authors generated initial boundary conditions for coupled fire-atmosphere simulations that assessed the fuel representation, using imagery, machine learning, and field sampling. In [53], high-resolution fuel moisture content and gridded data are developed, with the aim of assimilation into operational fire prediction (by establishing relationships between the satellite reflectance, surface weather and soil moisture observations, and fuel moisture content). Surface observations were used to train multiple machine learning methods (multiple linear regression, random forests (RFs), gradient boosted regression, and neural networks).

In [54], the authors combined multi-source remote sensing and field data with machine learning techniques (random forest and support vector machine) and traditional regression models, to estimate the dead fuel load in a $1 \mathrm{~h}$ time horizon, and to understand its determining factors. In [55], the authors developed models to estimate the fuel moisture content in a $10 \mathrm{~h}$ horizon, making use within their framework of random forest and support vector machine methods.

A mask region-based convolutional neural network approach was used to automate dead tree detection from aerial spaces in [56]. In [57], neural networks are used to classify the presence of wildfire-ignitable liquids on the ground. In [58], a similar approach is adopted, but instead using K-nearest neighbours and support vector machine.

The optimal timing and location of fuel treatments and timber harvesting to prevent wildfires is studied in [59], where the authors use approximate dynamic programming and account for the spatial interactions that generate fire risk.

\section{Risk Assessment and Ignition Prediction of Wildfires}

These applications assess both the likelihood of wildfire occurrence and the occurrence impacts [60], and are an important component of disaster and risk mitigation studies [61]. In [2], the authors used random undersampling and boosting to address the drivers of a wildfire occurrence and to determine its risk from the multidimensional perspective of human activity, topology and geography, and land coverage, to deliver findings that can be used for territorial planning. In [62], remote sensing and machine learning techniques were used to explore fire determinant features (topography and human-accessibility-related features) and also to predict the probability of fire occurrence and danger. Fire danger maps are studied in [63], but from an approach of evaluation of a land cover map and information from previous fire-affected areas, among other features.

In [64], the wildfire risk is analysed under climate change scenarios, by developing fire frequency predictions employing random forest, support vector machine and polynomial machine learning regression methods. In [65], maximum entropy and random forest methods were tested to determine the risk of wildfire based on satellite images at various spatial and spectral resolutions.

A forest fire susceptibility index and a social/infrastructural vulnerability index are developed in [66], employing machine learning methods and GIS multi-criteria decisionmaking, with a forest fire susceptibility map as an output. In [67], the authors define a long-term index for wildfire risk and wildfire danger assessment by employing a fuzzy K-nearest neighbours classification approach. In [68], logistic regression, deep neural network, and fire risk indexing models are used to develop an optimized risk indexing system for wildfire risk assessment. Anthropogenic features were also assessed in [69] via the application of machine learning models to predict forest fires. In [70], an hourly risk index is developed based on a CatBoost method. In [71], socio-economic factors are also considered to determine the wildfire probability using a maximum entropy method associated with a random forest method.

In [72], forest susceptibility risk is explored using a new ensemble model based on two deep neural networks, and the model is compared with methods such as extreme gradient boosting and support vector machine. Another susceptibility map is elaborated in [73], based on a random forest method that takes into consideration previous fire perimeters and various geo-environmental predisposing factors. In [74], forest fire susceptibility 
mapping was developed by employing a LogitBoost ensemble-based decision tree method and benchmarked against support vector machine, random forest, and kernel logistic regression. In [75], geospatial data, multiple machine learning (neural networks, support vector machine, and maximum entropy, among others), and spatial statistical tools are used to demarcate the susceptibility to fire in forests using a weighted approach. In [76], a machine learning ensemble approach (based on the prediction results of support vector machine and random forest) is developed to predict wildfires. In [77], the susceptibility assessment is performed via the random forest method.

In [78], the performance of many machine learning algorithms (naive Bayes, Bayes network, multivariate logistic regression, and decision tree) are tested for the prediction and development of a fire susceptibility map. The performance accuracy is also evaluated in [79], in which boosted regression tree, functional discriminant analysis, classification and regression trees, generalized linear model, random forest, mixture discriminant analysis, and a few hybrid methods are developed to predict wildfire hazard and wildfire-prone areas. In addition, in [80], a multi-hazard risk map (in which wildfires are included) is developed, and the approach (using support vector machine, boosted regression tree, and generalized linear model methods) focuses on the improvement of its accuracy. The improvement of fire risk mapping via satellite-derived metrics is studied in [81], in which logistic regression, random forest, and extreme gradient boosting are used. Forest susceptibility is mapped by new hybrid algorithms proposed in [82], and in [83] that susceptibility is assessed by employing a local weighted learning algorithm with cascade generalization. Forest hazards are also evaluated in [84], by employing an optimized repeatedly random undersampling method using support vector machine and a genetic algorithm to compute its parameters.

In [85], the wildfire occurrence is predicted by employing random forest algorithms and cluster analysis. The authors analyse changes in spatial patterns of ignition probability over time, taking into consideration human-related drivers, among others. In [86], the estimation of wildfire probability is achieved by employing neural networks and taking into consideration features related to biophysical and human drivers.

In [87], a combination of big data, remote sensing, and machine learning methods (neural networks and support vector machine) is used to extract insights from satellite images to model the prediction of the occurrence of wildfires. In [88], the authors use machine learning methods to assess the probability of a fire event starting within a $24 \mathrm{~h}$ horizon from lightning events. In [3], the authors use a deep neural network approach to estimate and predict wildfire ignition risk, mainly based on topological attributes. In [4], a GIS-aided maximum entropy method is used for the development of a fire prediction map, and a feature selection study is performed considering environmental features. In [89], a total of thirty-six features are evaluated to determine those that provide better performance of the prediction model. In [1], the authors used random forest models and an ensemble approach to predict wildfire risk. A convolutional neural network is used to deliver a spatial prediction model for forest fires in [90], using a set of GIS-based data. In [91], a GIS-based machine learning method is also developed, in which multiple machine learning methods are tested.

In [92], the authors employ a broad diversity of machine learning methods targeting the prediction of the occurrence of forest fires (namely, decision forest classifier, boosted decision trees, decision jungle classifier, averaged perceptron, local deep support vector machine, 2-class Bayes point machine, logistic regression, and binary neural network). Based on the comparison of results, the authors proposed an Internet-of-Things-based smart fire prediction system. In [93], detailed topological features are used in a hybrid artificial intelligence model (composed of multivariate adaptive regression splines optimized by differential flower pollination). In [94], the authors develop a comparison between the prediction of fire behaviour using machine learning and a physically based method, mainly evaluating the performance and computational time. The focus is on the calibration of wildfire prediction machine learning models in [60], in which the methods of random forests, neural networks, and classification trees are the objects of study, in comparison with 
traditional methods such as logistic regression and logistic generalized additive models. Random forest is also used in [95].

In [96], the probability of wildfire is modelled based on a machine learning approach, and it is analysed in terms of the costs associated with the implementation of multiple prescriptions for risk mitigation. In [97], the authors assess, by means of employing gradient boosting tree, the impact of weather on the damage caused by fire incidents and also predict its risk. In [98], a model is proposed to predict fire risk one month in advance.

\section{Support to Dispatch}

The work presented in [99] aims at supporting wildfire planning management activities by establishing road interrelations between wildfire operational delineations and potential control locations, using boosted regression models to facilitate future dispatch of suppression resources. In [100], work is also focused on the firefighters dispatch strategy, by assessing the probability of fire containment. The models used as training data georeferenced historical fire data concerning ignition locations, previous responses, and weather conditions. They assessed different gradients of features such as detection time, ground accessibility, and aerial support.

Landscape Planning and Prevention Measures for Severity Mitigation

In [48], the authors assess the delineation of the wildland-urban interface based on wildfire risk assessment, with a focus on whether fire-based machine learning mapping enhances the spatial congruence of houses and wildfires, compared with conventional methods. In [101], the authors study the landslide and wildfire intersection susceptibility, taking into consideration the uncertainty of susceptibility maps by using three advanced ensemble machine learning algorithms: adaptive boosting, random forest and gradient boosting decision tree. In [102], four machine learning classifiers were applied to establish the wildland-urban interface definition based on fire occurrence data, focusing on housing density and vegetation coverage. Finally, in [103], the impacts of prescribed burning treatments are assessed using the random forest method, with the aim of reducing extreme wildfires. Eight features were evaluated, among them the fire return interval, the seasonality of the burn, and hydrological and climatic variables.

Table 2. Results: pre-fire prevention applications.

\begin{tabular}{|c|c|c|c|c|c|}
\hline Ref. & Classification $^{1}$ & Detailed Application & $\begin{array}{l}\text { Machine Learning } \\
\text { Technique }\end{array}$ & $\begin{array}{l}\text { Case Study } \\
\text { Location }\end{array}$ & $\begin{array}{l}\text { Model Performance } \\
\text { Metrics Results }\end{array}$ \\
\hline [51] & $\mathrm{A}$ & $\begin{array}{l}\text { Methods of Fuel } \\
\text { Management Zone } \\
\text { improvement }\end{array}$ & $\begin{array}{l}\text { Extreme Gradient Boosting, } \\
\text { Support Vector Machines, } \\
\text { K-Nearest Neighbours, and } \\
\text { Random Forest. }\end{array}$ & & $\begin{array}{c}\text { F1-score ranging from } \\
90.0 \% \text { up to } 94.0 \% \text { and } \\
\text { a Kappa ranging from } \\
0.80 \text { up to } 0.89 .\end{array}$ \\
\hline [53] & A & $\begin{array}{l}\text { Determining fuel } \\
\text { moisture content }\end{array}$ & $\begin{array}{l}\text { Multiple Linear Regression, } \\
\text { Random Forests, Gradient } \\
\text { Boosted Regression, and } \\
\text { Neural Networks }\end{array}$ & United States & $\begin{array}{l}\text { Errors between } \\
25.0-33.0 \%\end{array}$ \\
\hline [54] & A & $\begin{array}{l}\text { Estimating fine dead } \\
\text { fuel load and } \\
\text { understand its } \\
\text { determining factors }\end{array}$ & $\begin{array}{l}\text { Multiple Linear Regression, } \\
\text { Random Forest, and } \\
\text { Support Vector Machine }\end{array}$ & & $\begin{array}{c}\text { Random Forest: RMSE: } \\
0.09 \text {; MSE: } 0.01 \text {; r: } 0.71 ; \\
\text { R-2: 0.50) }\end{array}$ \\
\hline [55] & A & $\begin{array}{l}\text { Estimating } 10 \mathrm{~h} \text { fuel } \\
\text { moisture content }\end{array}$ & $\begin{array}{l}\text { Random Forest and Support } \\
\text { Vector Machine }\end{array}$ & & $\begin{array}{c}(\mathrm{R}-2=0.77-0.82 \text { and } \\
\text { root mean squared } \\
\text { error }[\mathrm{RMSE}]= \\
2.0-2.8 \%)\end{array}$ \\
\hline
\end{tabular}


Table 2. Cont.

\begin{tabular}{|c|c|c|c|c|c|}
\hline Ref. & Classification $^{1}$ & Detailed Application & $\begin{array}{l}\text { Machine Learning } \\
\text { Technique }\end{array}$ & $\begin{array}{l}\text { Case Study } \\
\text { Location }\end{array}$ & $\begin{array}{l}\text { Model Performance } \\
\text { Metrics Results }\end{array}$ \\
\hline [56] & A & $\begin{array}{l}\text { Detection of dead trees } \\
\text { from aerial images }\end{array}$ & $\begin{array}{c}\text { Mask Region-Based } \\
\text { Convolutional Neural } \\
\text { Network }\end{array}$ & & $\begin{array}{l}\text { Mean average precision } \\
\text { score }=54.0 \%\end{array}$ \\
\hline [57] & A & $\begin{array}{c}\text { Detection of ignitable } \\
\text { liquids on } \\
\text { ground-truth fire } \\
\text { debris }\end{array}$ & Neural Networks & & $\begin{array}{c}\text { False positive rate of } \\
0.07 \text { and a true positive } \\
\text { rate of } 0.59\end{array}$ \\
\hline [58] & $\mathrm{A}$ & $\begin{array}{l}\text { Detection of ignitable } \\
\text { liquid residue on fire } \\
\text { debris }\end{array}$ & $\begin{array}{l}\text { Linear and Quadratic } \\
\text { Discriminant Analysis, } \\
\text { K-Nearest Neighbours, and } \\
\text { Support Vector Machines } \\
\text { with Radial and Linear } \\
\text { Kernels }\end{array}$ & & $\begin{array}{l}\text { Area under the receiver } \\
\text { operating characteristic } \\
\text { curve }(0.86-0.92), \text { Equal } \\
\text { error rates }(17.0-22.0 \%)\end{array}$ \\
\hline [60] & B & $\begin{array}{c}\text { Methods for properly } \\
\text { calibrating statistical } \\
\text { and machine learning } \\
\text { models for fine-scale, } \\
\text { spatially explicit daily } \\
\text { fire occurrence } \\
\text { prediction }\end{array}$ & $\begin{array}{l}\text { Classification Trees, } \\
\text { Random Forests, Neural } \\
\text { Networks, Logistic } \\
\text { Regression Models, and } \\
\text { Logistic Generalized } \\
\text { Additive Models }\end{array}$ & Alberta, Canada & \\
\hline [61] & B & $\begin{array}{l}\text { Monitoring fire risks } \\
\text { over a large region }\end{array}$ & Transductive PU Learning & Southeast China & $\begin{array}{l}\text { High sensitivity } \\
\quad(>80.0 \%)\end{array}$ \\
\hline [2] & B & $\begin{array}{l}\text { Addressing the } \\
\text { multidimensional } \\
\text { effects of three groups } \\
\text { of drivers in territorial } \\
\text { planning under fire } \\
\text { risk }\end{array}$ & $\begin{array}{l}\text { Random Undersampling } \\
\text { and Boosting }\end{array}$ & Chile & $\begin{array}{l}\text { Area under the receiver } \\
\text { operating characteristic } \\
\text { curve of } 0.967 \text { and an } \\
\text { overall accuracy over } \\
\text { test data of } 93.0 \%\end{array}$ \\
\hline [63] & B & Modelling fire danger & $\begin{array}{l}\text { Support Vector Machine, } \\
\text { Generalized Linear Model, } \\
\text { Functional Data Analysis, } \\
\text { and Random Forest }\end{array}$ & Iran & $\begin{array}{c}\text { Area under the receiver } \\
\text { operating characteristic } \\
\text { curve of } 0.855\end{array}$ \\
\hline [64] & B & $\begin{array}{l}\text { Analysing the } \\
\text { influences of climate } \\
\text { warming on fire risk }\end{array}$ & $\begin{array}{l}\text { Random Forest, Support } \\
\text { Vector Machine and } \\
\text { Polynomial }\end{array}$ & Changsha, China & \\
\hline [65] & B & $\begin{array}{l}\text { Determining the risk } \\
\text { of fire }\end{array}$ & Maxent and Random Forest & Yakutia, Russia & \\
\hline [66] & B & $\begin{array}{l}\text { Developing spatial } \\
\text { prediction of wildfire } \\
\text { susceptibility }\end{array}$ & $\begin{array}{l}\text { Artificial Neural Network, } \\
\text { Support Vector Machines, } \\
\text { and Random Forest }\end{array}$ & Iran & $\begin{array}{c}\text { Accuracies between } \\
74.0-88.0 \%\end{array}$ \\
\hline [67] & B & $\begin{array}{c}\text { Defining a long-term } \\
\text { wildfire warning index }\end{array}$ & $\begin{array}{l}\text { Fuzzy K-Nearest } \\
\text { Neighbours }\end{array}$ & Brazil & \\
\hline [68] & B & $\begin{array}{c}\text { Optimizing risk } \\
\text { indexing for fire risk } \\
\text { assessment }\end{array}$ & Deep Neural Networks & Korea & \\
\hline [69] & B & $\begin{array}{l}\text { Determining the main } \\
\text { explanatory variables } \\
\text { for forest fire } \\
\text { occurrence and } \\
\text { mapping of } \\
\text { probability }\end{array}$ & Random Forest & Eastern Serbia & \\
\hline
\end{tabular}


Table 2. Cont.

\begin{tabular}{|c|c|c|c|c|c|}
\hline Ref. & Classification $^{1}$ & Detailed Application & $\begin{array}{l}\text { Machine Learning } \\
\text { Technique }\end{array}$ & $\begin{array}{l}\text { Case Study } \\
\text { Location }\end{array}$ & $\begin{array}{l}\text { Model Performance } \\
\text { Metrics Results }\end{array}$ \\
\hline [70] & B & $\begin{array}{l}\text { Developing an hourly } \\
\text { forest fire risk index }\end{array}$ & CatBoost & South Korea & $\begin{array}{l}\text { Area under the receiver } \\
\text { operating characteristic } \\
\text { curve }=0.8434\end{array}$ \\
\hline [71] & B & $\begin{array}{l}\text { Estimating and } \\
\text { analysing how human } \\
\text { activity is influencing } \\
\text { forest fire probability }\end{array}$ & $\begin{array}{l}\text { Maximum Entropy (Maxent) } \\
\text { and Random Forest }\end{array}$ & South Korea & \\
\hline [72] & $\mathrm{B}$ & $\begin{array}{l}\text { Prediction of fire } \\
\text { susceptibility and } \\
\text { effects of sample patch } \\
\text { sizes on the predictive } \\
\text { performance of the } \\
\text { algorithms }\end{array}$ & Deep Neural Network & Chile & $\begin{array}{l}\text { Area under the } \\
\text { curve }=0.953\end{array}$ \\
\hline [73] & B & $\begin{array}{l}\text { Elaborating a wildfire } \\
\text { susceptibility map }\end{array}$ & Random Forests & Italy & \\
\hline [74] & B & $\begin{array}{l}\text { Developing a forest } \\
\text { fire susceptibility map }\end{array}$ & $\begin{array}{l}\text { LogitBoost Ensemble-Based } \\
\text { Decision Tree }\end{array}$ & Vietnam & $\begin{array}{l}92.0 \% \text { prediction } \\
\text { capability }\end{array}$ \\
\hline [75] & B & $\begin{array}{l}\text { Weighted approach to } \\
\text { characterizing the } \\
\text { forest fire } \\
\text { susceptibility }\end{array}$ & $\begin{array}{l}\text { Artificial Neural Network, } \\
\text { Generalized Linear Model, } \\
\text { Multivariate Adaptive } \\
\text { Regression Splines, Naive } \\
\text { Bayesian Classifier, } \\
\text { K-Nearest Neighbour, } \\
\text { Support Vector Machine, } \\
\text { Random Forest, Gradient } \\
\text { Boosting Machine, Adaptive } \\
\text { Boosting, and Maximum } \\
\text { Entropy (Maxent) }\end{array}$ & Kerala, India & $\begin{array}{c}\text { Receiver operating } \\
\text { characteristics-area } \\
\text { under curve values } \\
\text { ranging from } 0.869 \text { to } \\
0.924\end{array}$ \\
\hline [76] & B & $\begin{array}{l}\text { Generating } \\
\text { susceptibility maps of } \\
\text { forest fires }\end{array}$ & $\begin{array}{c}\text { Support Vector Machine, } \\
\text { Random Forest, and } \\
\text { Ensemble }\end{array}$ & Serbia & $\begin{array}{c}\text { Ensemble model had } \\
\text { an area under } \\
\text { curve }=0.848\end{array}$ \\
\hline [77] & B & $\begin{array}{l}\text { Developing a model, } \\
\text { in which probabilistic } \\
\text { outputs allowed } \\
\text { elaboration of wildfire } \\
\text { susceptibility maps. }\end{array}$ & Random Forest & Bolivia & \\
\hline [78] & $\mathrm{B}$ & $\begin{array}{l}\text { Prediction and } \\
\text { mapping of fire } \\
\text { susceptibility }\end{array}$ & $\begin{array}{l}\text { Bayes Network, Naive } \\
\text { Bayes, Decision Tree, and } \\
\text { Multivariate Logistic } \\
\text { Regression }\end{array}$ & $\begin{array}{l}\text { Pu Mat National } \\
\text { Park, Nghe An } \\
\text { Province, Vietnam }\end{array}$ & Area under curve $=0.96$ \\
\hline [79] & B & $\begin{array}{l}\text { Predicting the fire } \\
\text { hazard in a fire-prone } \\
\text { area }\end{array}$ & $\begin{array}{l}\text { Boosted Regression Tree, } \\
\text { Classification and } \\
\text { Regression Trees, Functional } \\
\text { Discriminant Analysis, } \\
\text { Generalized Linear Model, } \\
\text { Mixture Discriminant } \\
\text { Analysis, Random Forest }\end{array}$ & $\begin{array}{l}\text { Northeast Iran, } \\
\text { Golestan } \\
\text { Province. }\end{array}$ & $\begin{array}{c}\text { Area under } \\
\text { curve }=0.855\end{array}$ \\
\hline [80] & B & $\begin{array}{c}\text { Producing an accurate } \\
\text { multi-hazard risk map } \\
\text { for a mountainous } \\
\text { area }\end{array}$ & $\begin{array}{c}\text { Support Vector Machine, } \\
\text { Boosted Regression Tree, } \\
\text { and Generalized Linear } \\
\text { Model }\end{array}$ & $\begin{array}{l}\text { Mountainous } \\
\text { region of Iran }\end{array}$ & \\
\hline
\end{tabular}


Table 2. Cont.

\begin{tabular}{|c|c|c|c|c|c|}
\hline Ref. & Classification $^{1}$ & Detailed Application & $\begin{array}{l}\text { Machine Learning } \\
\text { Technique }\end{array}$ & $\begin{array}{l}\text { Case Study } \\
\text { Location }\end{array}$ & $\begin{array}{l}\text { Model Performance } \\
\text { Metrics Results }\end{array}$ \\
\hline [81] & B & $\begin{array}{l}\text { Investigating the } \\
\text { impact of } \\
\text { satellite-derived } \\
\text { metrics that represent } \\
\text { long-term vegetation } \\
\text { status and dynamics } \\
\text { on fire risk mapping }\end{array}$ & $\begin{array}{c}\text { Logistic Regression, } \\
\text { Random Forest, and } \\
\text { Extreme Gradient Boosting }\end{array}$ & $\begin{array}{l}\text { Mediterranean } \\
\text { woodlands and } \\
\text { forests }\end{array}$ & \\
\hline [82] & B & $\begin{array}{l}\text { Mapping forest fire } \\
\text { susceptibility }\end{array}$ & $\begin{array}{c}\text { Frequency Ratio-Multilayer } \\
\text { Perceptron, Frequency } \\
\text { Ratio-Logistic Regression, } \\
\text { Frequency } \\
\text { Ratio-Classification and } \\
\text { Regression Tree, Frequency } \\
\text { Ratio-Support Vector } \\
\text { Machine, and Frequency } \\
\text { Ratio-Random Forest }\end{array}$ & North Morocco & $\begin{array}{l}\text { Area under } \\
\text { curve }=0.989\end{array}$ \\
\hline [83] & B & $\begin{array}{l}\text { Prediction of forest fire } \\
\text { susceptibility }\end{array}$ & $\begin{array}{l}\text { Locally Weighted Learning } \\
\text { Algorithm with the Cascade } \\
\text { Generalization, Bagging, } \\
\text { Decorate, and Dagging } \\
\text { Ensemble Learning }\end{array}$ & Vietnam & $\begin{array}{l}\text { Area under } \\
\text { curve }=0.993\end{array}$ \\
\hline$[84]$ & B & $\begin{array}{c}\text { Computing the } \\
\text { probability of hazard } \\
\text { occurrence }\end{array}$ & $\begin{array}{l}\text { Support Vector Machine and } \\
\text { Genetic Algorithm }\end{array}$ & & \\
\hline [85] & B & $\begin{array}{l}\text { Predicting and } \\
\text { detecting changes in } \\
\text { the spatial pattern of } \\
\text { ignition probability } \\
\text { over time. }\end{array}$ & Random Forest & Brazil & $\begin{array}{l}\text { Area under } \\
\text { curve }=0.72\end{array}$ \\
\hline [86] & B & $\begin{array}{l}\text { Estimating wildfire } \\
\text { probability occurrence } \\
\text { as a function of } \\
\text { biophysical and } \\
\text { human-related drivers }\end{array}$ & Artificial Neural Network & $\begin{array}{c}\text { Alpine and } \\
\text { subalpine region }\end{array}$ & $\begin{array}{c}\text { Area under } \\
\text { curve }=0.68-0.72\end{array}$ \\
\hline [87] & B & $\begin{array}{c}\text { Predicting the } \\
\text { occurrence of wildfires }\end{array}$ & $\begin{array}{l}\text { Artificial Neural Network } \\
\text { and Support Vector Machine }\end{array}$ & & $\begin{array}{c}\text { Prediction } \\
\text { accuracy }=98.3 \%\end{array}$ \\
\hline [3] & B & $\begin{array}{l}\text { Estimating and } \\
\text { predicting wildfire } \\
\text { ignition risk }\end{array}$ & Deep Neural Network & & \\
\hline [4] & B & $\begin{array}{l}\text { Proposing forest fire } \\
\text { prediction map }\end{array}$ & Maximum Entropy & $\begin{array}{l}\text { Brazil and } \\
\text { Australia }\end{array}$ & Area under curve $=0.95$ \\
\hline$[1]$ & B & $\begin{array}{l}\text { Predicting the wildfire } \\
\text { risk }\end{array}$ & Random Forest & $\begin{array}{c}\text { Monticello and } \\
\text { Winters, } \\
\text { California }\end{array}$ & Accuracy of $92.0 \%$ \\
\hline [90] & B & $\begin{array}{l}\text { Proposing a spatial } \\
\text { prediction model for } \\
\text { forest fire } \\
\text { susceptibility }\end{array}$ & $\begin{array}{c}\text { Convolutional Neural } \\
\text { Network }\end{array}$ & $\begin{array}{l}\text { Yunnan Province, } \\
\text { China }\end{array}$ & Area under curve $=0.86$ \\
\hline [91] & B & $\begin{array}{l}\text { Assessing forest fire } \\
\text { susceptibility }\end{array}$ & $\begin{array}{l}\text { Boosted Regression Tree, } \\
\text { General Linear Model, and } \\
\text { Mixture Discriminant } \\
\text { Analysis }\end{array}$ & $\begin{array}{l}\text { Fars Province, } \\
\text { Iran }\end{array}$ & \\
\hline
\end{tabular}


Table 2. Cont.

\begin{tabular}{|c|c|c|c|c|c|}
\hline Ref. & Classification $^{1}$ & Detailed Application & $\begin{array}{c}\text { Machine Learning } \\
\text { Technique }\end{array}$ & $\begin{array}{l}\text { Case Study } \\
\text { Location }\end{array}$ & $\begin{array}{l}\text { Model Performance } \\
\text { Metrics Results }\end{array}$ \\
\hline [92] & B & $\begin{array}{l}\text { Predicting occurrence } \\
\text { of forest fires }\end{array}$ & $\begin{array}{l}\text { Boosted Decision Trees, } \\
\text { Decision Forest Classifier, } \\
\text { Decision Jungle Classifier, } \\
\text { Averaged Perceptron, } \\
\text { 2-Class Bayes Point } \\
\text { Machine, Local Deep } \\
\text { Support Vector Machine, } \\
\text { Logistic Regression, and } \\
\text { Binary Neural Network }\end{array}$ & & $\begin{array}{l}\text { Area under the } \\
\text { curve }=0.78\end{array}$ \\
\hline [93] & B & $\begin{array}{c}\text { Analysing and } \\
\text { predicting spatial } \\
\text { patterns of forest fire } \\
\text { danger }\end{array}$ & $\begin{array}{l}\text { Multivariate Adaptive } \\
\text { Regression Splines } \\
\text { Optimized by Differential } \\
\text { Flower Pollination }\end{array}$ & $\begin{array}{l}\text { Lao Cai province } \\
\text { (Vietnam) }\end{array}$ & $\begin{array}{l}\text { Area under the } \\
\text { curve }=0.91\end{array}$ \\
\hline [94] & B & $\begin{array}{l}\text { Providing details on } \\
\text { specific techniques } \\
\text { being explored for } \\
\text { performing low-cost, } \\
\text { high fidelity fire } \\
\text { predictions }\end{array}$ & Deep Neural Networks & & \\
\hline [60] & B & $\begin{array}{l}\text { Developing methods } \\
\text { for properly } \\
\text { calibrating statistical } \\
\text { and machine learning } \\
\text { models for fine-scale, } \\
\text { spatially explicit daily } \\
\text { predictions }\end{array}$ & $\begin{array}{l}\text { Classification Trees, } \\
\text { Random Forests, Neural } \\
\text { Networks, Logistic } \\
\text { Regression Models, and } \\
\text { Logistic Generalized } \\
\text { Additive Models }\end{array}$ & $\begin{array}{l}\text { Lac La Biche } \\
\text { region of Alberta, } \\
\text { Canada }\end{array}$ & \\
\hline [95] & B & $\begin{array}{l}\text { Estimating the } \\
\text { probability of fire } \\
\text { occurrence }\end{array}$ & Random Forest & $\begin{array}{l}\text { Colombian- } \\
\text { Venezuelan plains } \\
\text { (llanos) ecoregion } \\
\text { in South America. }\end{array}$ & Accuracy of $94.0 \%$ \\
\hline [97] & $\mathrm{B}$ & $\begin{array}{l}\text { Studying the impact of } \\
\text { weather on the } \\
\text { damage caused by fire } \\
\text { incidents }\end{array}$ & Gradient Boosting Tree & United States & $\begin{array}{c}\text { R-2 value of } 0.933 \text { and } \\
\text { mean squared error } \\
\text { (MSE) of } 124.641 \text { out of } \\
10,000\end{array}$ \\
\hline [98] & B & $\begin{array}{l}\text { Prediction of African } \\
\text { fire one month in } \\
\text { advance and } \\
\text { generalizing to } \\
\text { provide seasonal } \\
\text { estimates of regional } \\
\text { and global fire risk }\end{array}$ & $\begin{array}{c}\text { Stepwise Generalized } \\
\text { Equilibrium Feedback } \\
\text { Assessment }\end{array}$ & Africa & \\
\hline [101] & $\mathrm{D}$ & $\begin{array}{c}\text { Developing } \\
\text { susceptibility maps } \\
\text { considering the } \\
\text { intersection of } \\
\text { landslide and wildfire } \\
\text { susceptibility and the } \\
\text { spatial uncertainty }\end{array}$ & $\begin{array}{l}\text { Random Forest, Gradient } \\
\text { Boosting Decision Tree, and } \\
\text { Adaptive Boosting }\end{array}$ & & \\
\hline
\end{tabular}


Table 2. Cont.

\begin{tabular}{|c|c|c|c|c|c|}
\hline Ref. & Classification $^{1}$ & Detailed Application & $\begin{array}{c}\text { Machine Learning } \\
\text { Technique }\end{array}$ & $\begin{array}{l}\text { Case Study } \\
\text { Location }\end{array}$ & $\begin{array}{l}\text { Model Performance } \\
\text { Metrics Results }\end{array}$ \\
\hline [104] & $\mathrm{E}$ & $\begin{array}{l}\text { Creating wildfire } \\
\text { inventory data by } \\
\text { integrating the } \\
\text { polygons collected } \\
\text { through field surveys } \\
\text { using global } \\
\text { positioning systems } \\
\text { (GPS) and the data } \\
\text { collected from the } \\
\text { moderate resolution } \\
\text { imaging spectrometer } \\
\text { (MODIS) thermal } \\
\text { anomalies product }\end{array}$ & $\begin{array}{l}\text { Artificial Neural Network, } \\
\text { Dmine Regression, DM } \\
\text { Neural, Least Angle } \\
\text { Regression, Multi-Layer } \\
\text { Perceptron, Random Forest, } \\
\text { Radial Basis Function, } \\
\text { Self-Organizing Maps, } \\
\text { Support Vector Machine, } \\
\text { and Decision Tree }\end{array}$ & & \\
\hline [105] & $\mathrm{E}$ & $\begin{array}{c}\text { Developing an } \\
\text { automatized and } \\
\text { cloud-based workflow } \\
\text { for generating a } \\
\text { training dataset of fire } \\
\text { events at a continental } \\
\text { level using freely } \\
\text { available remote } \\
\text { sensing data }\end{array}$ & $\begin{array}{l}\text { Random Forest, Naive } \\
\text { Bayes, and Classification } \\
\text { and Regression Tree. }\end{array}$ & & \\
\hline [106] & $\mathrm{E}$ & $\begin{array}{l}\text { Creating a wildfire } \\
\text { data inventory by } \\
\text { integrating global } \\
\text { positioning system } \\
\text { (GPS) polygons with } \\
\text { data collected from the } \\
\text { moderate resolution } \\
\text { imaging } \\
\text { spectroradiometer } \\
\text { (MODIS) thermal } \\
\text { anomalies product }\end{array}$ & $\begin{array}{l}\text { Artificial Neural Network, } \\
\text { Support Vector Machines, } \\
\text { and Random Forest }\end{array}$ & $\begin{array}{l}\text { Amol County, } \\
\text { northern Iran. }\end{array}$ & \\
\hline
\end{tabular}

${ }^{1}$ References were classified according to the application focus: (A) wildfire fuel modelling; (B) risk assessment and ignition prediction of wildfires; (D) landscape planning and prevention measures for severity mitigation; (E) development of inventory data.

\section{Development of Inventory Data}

In [104], the wildfire susceptibility was assessed and inventory data were developed by the integration of GPS and MODIS thermal anomalies product data. Along with the developed inventory data, conditioning factors were selected and tested with ten different machine learning methods and compared against the traditional logistic regression method. In [105], the authors create inventory data for training machine learning models of fire events using freely available remote sensing data. The training dataset generated was applied in random forest, classification and regression tree, and naïve Bayes methods. In [106], a wildfire and conditioning factors data inventory is proposed by also integrating GPS and MODIS data. The authors demonstrate the application of neural networks, support vector machine, and random forest to the data.

Table 2 presents in more details the classification of the mentioned articles considering the pre-fire prevention applications.

\subsubsection{Management of Active Wildfires (Detection and Response)}

The 21 papers addressing this wildfire cycle management phase focused on the following applications: wildfire detection, wildfire spread prediction, and wildfire suppression. 
Of the 21 articles, $61.9 \%$ applied machine learning to wildfire detection (A), $23.8 \%$ to wildfire spread prediction (B), and $14.3 \%$ to wildfire suppression (C).

\section{Wildfire Detection}

The ability to rapidly detect the ignition of a wildfire is of paramount importance in order to avoid the wildfire turning into an extreme wildfire event [107]. There is thus an urgent need to focus on high-performance forest fire detection models. Wildfire detection models should ideally have high fire detection rate and a low false alarm rate [108].

A machine learning image-based model prototype was developed in [109], to detect smoke from fires within 15 min of ignition. In [110], an image-based smoke detection method is designed, based on a convolutional neural network approach that works as a binary classifier. In [111], an imagery-based detection model is presented, and a decision rule and a Gaussian mixture model are employed to train the model. The authors in [112] also use imagery-based fire detection, associated with the random forest method to reduce false alarms. In [113], image features are used alongside a long short term memory network and a convolutional neural network. Deep learning is used in a multi-level forest detection method for wildfires in [114]. In [115], an adaptive quasi-unsupervised approach is used to monitor the boundary conditions to detect forest fires.

A multi-sensor machine-learning-based system is developed in [116], based also on neural networks. In [117], a similar approach is used but the focus is on surveillance camera use. A fire-flake generator model based on ambient features and machine learning is developed in [118]. In [119], PRISMA sensor data were associated with classification techniques from the support vector machine method.

In [108], the authors aim to increase the accuracy of detection and reduce false alarms by using a K-nearest neighbour method. In [120], powerlines are used associated with extreme gradient boosting machine learning to detect wildfires. In [61], the authors used transductive learning from the positive and unlabelled (PU learning) data method to identify forest fire occurrence, strongly based on remote sensing data. In [121], satellite images are used to identify forest fires, based on a random forest method.

\section{Wildfire Spread Prediction}

In [122], an in-time methodology is developed both to optimize effective fire containment resource utilization and (mainly) to predict fire spread, through the use of an ensemble model based on machine learning methods (e.g., particle swarm and a bat algorithm), a heuristic approach and principal component regression. Short-term spread prediction is developed in [19], with real-time rate-of-spread measurements associated with a machine learning algorithm for correlation. In [123], the fire arrival time is estimated based on satellite data and the support vector machine.

In [124], the fire front spread is estimated using a deep convolutional inverse graphics network. In [125], the prediction of wildfire spread is evaluated by employing a statistical downscaling scheme based on deep learning associated with multi-source remote sensing data. In [126], the drivers for the fire spread predicted severity as well as prescriptions are evaluated, associating a random forest method with remote sensing data. In [127], biophysical and management drivers of the final estimated severity of spread are evaluated through the use of the random forest method. Final fire size is predicted at the time of ignition in [128], using decision trees. In [129], machine learning algorithms are integrated with multistage fire spread models. In [107], there is a special focus on developing inventory data to characterize the possibility of a wildfire becoming an extreme wildfire event. The results are applied to train four machine learning methods. The authors concluded that the use of data registered at the time of the wildfire contributes to increasing the accuracy in predicting the probability of a wildfire becoming an extreme wildfire event, compared to the use of historical data. 


\section{Wildfire Suppression}

In [130], the authors develop a digital twin framework that combines the track of the trajectory of released airborne materials for fire suppression and machine learning to optimize the release dynamics of the aircraft in a fast manner. In [131], the research assesses a sound-wave fire-extinguishing system, by means of applying machine learning methods (neural networks, deep neural networks, random forest, K-nearest neighbour, and ensemble methods) to the dataset, to also classify extinction and non-extinction states of the fire.

Table 3. Results: management of active wildfire applications.

\begin{tabular}{|c|c|c|c|c|c|}
\hline Ref. & Classification $^{1}$ & Detailed Application & $\begin{array}{l}\text { Machine Learning } \\
\text { Technique }\end{array}$ & $\begin{array}{l}\text { Case Study } \\
\text { Location }\end{array}$ & $\begin{array}{c}\text { Model } \\
\text { Performance } \\
\text { Metrics Results }\end{array}$ \\
\hline [110] & A & $\begin{array}{l}\text { Proposing a two-module } \\
\text { video smoke detection } \\
\text { framework designed for } \\
\text { embedded applications on } \\
\text { local cameras }\end{array}$ & $\begin{array}{c}\text { Lightweight Deep } \\
\text { Convolutional Neural } \\
\text { Network }\end{array}$ & & \\
\hline [111] & $\mathrm{A}$ & $\begin{array}{l}\text { Proposing an intelligent fire } \\
\text { detection method by } \\
\text { investigating three } \\
\text { approaches to detect fire } \\
\text { based on three different } \\
\text { colour models }\end{array}$ & $\begin{array}{c}\text { Decision Rule and } \\
\text { Gaussian Mixture Model }\end{array}$ & & \\
\hline [112] & A & $\begin{array}{l}\text { Proposing a combined 3-step } \\
\text { forest fire detection algorithm } \\
\text { (i.e., thresholding, } \\
\text { machine-learning-based } \\
\text { modelling, and } \\
\text { post-processing) }\end{array}$ & Random Forest & South Korea & $\begin{array}{l}\text { Overall accuracy } \\
\text { similar to } 99.2 \%, \\
\text { probability of } \\
\text { detection }\end{array}$ \\
\hline [113] & $\mathrm{A}$ & $\begin{array}{l}\text { Proposing a multistage fire } \\
\text { detection method }\end{array}$ & $\begin{array}{l}\text { Convolutional Neural } \\
\text { Networks and Long } \\
\text { Short Term Memory } \\
\text { Networks }\end{array}$ & & \\
\hline [114] & A & $\begin{array}{l}\text { Proposing a multi-level forest } \\
\text { fire detection method }\end{array}$ & $\begin{array}{l}\text { General Advanced } \\
\text { Networks, Adaptive } \\
\text { Boosting, Convolutional } \\
\text { Neural Networks, and } \\
\text { Support Vector Machine }\end{array}$ & & \\
\hline [116] & A & $\begin{array}{l}\text { Proposing a method using } \\
\text { machine learning techniques } \\
\text { for multimedia surveillance } \\
\text { during fire emergencies }\end{array}$ & $\begin{array}{l}\text { Adaptive Boosting and } \\
\text { Many Multi-Layer } \\
\text { Perceptron Neural } \\
\text { Networks }\end{array}$ & & \\
\hline [117] & A & $\begin{array}{l}\text { Proposing a fire detection } \\
\text { method using sensors and } \\
\text { image data }\end{array}$ & $\begin{array}{c}\text { Adaptive Boosting, } \\
\text { Multi-Layer Perceptron } \\
\text { Neural Networks, and } \\
\text { Convolutional Neural } \\
\text { Networks }\end{array}$ & & \\
\hline [118] & A & $\begin{array}{l}\text { Proposing a data-driven } \\
\text { fire-flake simulation model }\end{array}$ & Neural Network & & \\
\hline [119] & A & $\begin{array}{l}\text { Exploring the potential use of } \\
\text { the PRISMA sensor for active } \\
\text { wildfire characterization }\end{array}$ & Support Vector Machine & $\begin{array}{l}\text { New South } \\
\text { Wales }\end{array}$ & \\
\hline
\end{tabular}


Table 3. Cont.

\begin{tabular}{|c|c|c|c|c|c|}
\hline Ref. & Classification $^{1}$ & Detailed Application & $\begin{array}{l}\text { Machine Learning } \\
\text { Technique }\end{array}$ & $\begin{array}{l}\text { Case Study } \\
\text { Location }\end{array}$ & $\begin{array}{c}\text { Model } \\
\text { Performance } \\
\text { Metrics Results }\end{array}$ \\
\hline [108] & A & $\begin{array}{l}\text { Proposing a method that } \\
\text { widens the view of fire } \\
\text { detection from conventional } \\
\text { two-class to multi-class } \\
\text { classification problems to } \\
\text { meet complex forest image } \\
\text { background }\end{array}$ & $\begin{array}{l}\text { K-Nearest Neighbour } \\
\text { Decision Tree }\end{array}$ & & \\
\hline [120] & A & $\begin{array}{l}\text { Exploring and discovering the } \\
\text { numerical patterns from the } \\
\text { contact to the ignition process } \\
\text { between different } \\
\text { upper-storey vegetations and } \\
\text { the powerlines }\end{array}$ & $\begin{array}{l}\text { Hybrid Step Extreme } \\
\text { Gradient Boosting }\end{array}$ & & \\
\hline$[61]$ & A & $\begin{array}{c}\text { Developing a workflow } \\
\text { process to monitor fires over a } \\
\text { large region }\end{array}$ & $\begin{array}{l}\text { Transductive PU } \\
\text { Learning }\end{array}$ & Southeast China & \\
\hline [121] & A & $\begin{array}{l}\text { Systematically testing and } \\
\text { comparing reflectance and } \\
\text { fractional cover candidate } \\
\text { severity indices }\end{array}$ & Random Forest & & \\
\hline [123] & B & $\begin{array}{l}\text { Estimating the fire arrival } \\
\text { time from satellite data }\end{array}$ & Support Vector Machine & $\begin{array}{l}\text { California, } \\
\text { United States }\end{array}$ & $\begin{array}{c}12.0 \% \text { burned area } \\
\text { absolute percentage } \\
\text { error; } 5.0 \% \text { total } \\
\text { burned area mean } \\
\text { percentage error, a } \\
0.21 \text { false alarm } \\
\text { ratio average, a } 0.86 \\
\text { probability of } \\
\text { detection average, } \\
\text { and a } 0.82 \\
\text { Sorensen's } \\
\text { coefficient average }\end{array}$ \\
\hline [124] & B & $\begin{array}{l}\text { Estimating the time-resolved } \\
\text { spatial evolution of a } \\
\text { wildland fire front }\end{array}$ & $\begin{array}{l}\text { Deep Convolutional } \\
\text { Inverse Graphics } \\
\text { Network }\end{array}$ & & \\
\hline [125] & B & $\begin{array}{l}\text { Developing a fire progression } \\
\text { model considering the } \\
\text { uncertainties }\end{array}$ & $\begin{array}{l}\text { U-Net Convolutional } \\
\text { Neural Network }\end{array}$ & & \\
\hline [126] & B & $\begin{array}{l}\text { Identifying the main } \\
\text { environmental factors driving } \\
\text { fire severity in extreme fire } \\
\text { events }\end{array}$ & Random Forest & California, USA & \\
\hline [128] & B & $\begin{array}{l}\text { Investigating the controls and } \\
\text { predictability of final fire size } \\
\text { at the time of ignition }\end{array}$ & Decision Tree & & $\begin{array}{l}50.4+/-5.2 \% \\
\quad \text { accuracy }\end{array}$ \\
\hline [131] & $\mathrm{C}$ & $\begin{array}{l}\text { Creating a sound-wave } \\
\text { fire-extinguishing system and } \\
\text { performing firefighting tests }\end{array}$ & $\begin{array}{c}\text { Artificial Neural } \\
\text { Network, K-Nearest } \\
\text { Neighbour, Random } \\
\text { Forest, Stacking, and } \\
\text { Deep Neural Network } \\
\text { Methods }\end{array}$ & & \\
\hline
\end{tabular}


Table 3. Cont.

\begin{tabular}{|c|c|c|c|c|c|}
\hline Ref. & Classification $^{1}$ & Detailed Application & $\begin{array}{c}\text { Machine Learning } \\
\text { Technique }\end{array}$ & $\begin{array}{l}\text { Case Study } \\
\text { Location }\end{array}$ & $\begin{array}{c}\text { Model } \\
\text { Performance } \\
\text { Metrics Results }\end{array}$ \\
\hline$[132]$ & C & $\begin{array}{l}\text { Developing a sound-wave } \\
\text { flame extinction system in } \\
\text { order to extinguish the flames } \\
\text { at an early stage of the fire }\end{array}$ & $\begin{array}{c}\text { Adaptive-Network- } \\
\text { Based Fuzzy Inference } \\
\text { Systems), CN2 Rule and } \\
\text { DT (Decision Tree) }\end{array}$ & & \\
\hline$[133]$ & C & $\begin{array}{l}\text { Creating an automated } \\
\text { system that is capable of } \\
\text { real-time, intelligent object } \\
\text { detection and recognition and } \\
\text { facilitating the improved } \\
\text { situational awareness of } \\
\text { firefighters during an } \\
\text { emergency response }\end{array}$ & $\begin{array}{c}\text { Convolutional Neural } \\
\text { Network }\end{array}$ & & \\
\hline
\end{tabular}

In [132], the authors develop further a sound-wave flame extinction system to target the wildfire at a very early stage. It is based on a developed dataset that considers features such as fuel type, flame size, decibels, frequency, airflow, and distance. The approach employs adaptive-network-based fuzzy inference systems and decision tree methods. In [133], the authors propose an automated real-time system of intelligent object detection and recognition to improve the situational awareness of firefighters during wildfire emergency response, using convolutional neural networks to classify and identify objects of interest from thermal imagery.

Table 3 presents in more details the classification of the mentioned articles considering the management of active wildfire applications.

\subsubsection{Post-Fire Wildfires (Restoration and Adaptation Activities)}

The evaluation of wildfire severity in a given area is of importance, as it allows us to estimate the economic impacts of the wildfire [134]. It further provides information to support restoration decisions and the prioritization of post-fire management strategies $[135,136]$. The 34 papers addressing this wildfire cycle management phase focused on the following applications: burned area and severity, impacts related to social factors, impacts related to carbon fluxes, and impacts related to forest conditions. Of the 34 articles, $61.8 \%$ applied machine learning to the characterization of burned area and severity (A), $8.8 \%$ to the assessment of impacts related to social factors (B), 5.9\% to the assessment of impacts related to carbon fluxes (C), and $23.5 \%$ to the assessment of impacts related to forest conditions (D).

\section{Burned Area and Severity}

Socio-economic features are evaluated in [137] along with meteorological and land surface characteristics, and the Shapley additive explanation method is used to predict the burned area. In [138], the authors assess the degree to which the machine learning training data affect the classification accuracy of fire severity modelling prediction. They consider the sample size and sample imbalance, also assessing the transferability of models to different geographic regions. In [139], random forest machine learning was used to determine the influence of pre-fire vegetation structure, weather conditions, and fire history on the wildfire severity, and to deliver management recommendations to mitigate the damage. In [140], an ensemble of machine learning methods approach is employed to predict the burned area. In [134], prediction of the severity is achieved using a combination of classification and regression machine learning algorithms. 
In [141], the authors used machine learning methods to estimate the burned area after a wildfire based on environmental factors such as fuel moisture, precipitation, vapour pressure, and temporal scale (dataset size). In [142], four hybrid models are compared to map wildland fire effects, using support vector machine and an adaptive neuro-fuzzy influence system, as well as meta-heuristic models. In [143], one of the modelling stages encompasses the processing of thematic maps of the area burnt, using machine learning techniques. In [136], automatic mapping of burned areas is achieved through the use of deep learning, considering a variety of network architectures applied to satellite data. The authors in [144] used pre and post-fire satellite images to identify the burn severity, employing random forest and support vector machine supervised classification. In [145], random forest also is used. Satellite imagery is used in [146], coupled with a random forest algorithm, both to detect recently burned areas and also to estimate the fire history.

In [147], remote sensing and machine learning are used to characterize burned areas in order to generate a map. Burned area detection for large areas is explored in [148], by means of satellite imagery and machine learning methods. In [149], the burned area is detected by evaluating the canopy cover pre- and post-fire, according to classification performed via machine learning. In another study, [150], a mask region-based convolutional neural network was used, coupled with a support vector machine to determine the post-fire affected areas, including pixels under the trees, based on images of the tree crowns. In [151], this detection is achieved through the analysis of reflectance contrasts associated with a classification regression tree algorithm. In [152-154], satellite images and the random forest machine learning method were used for the same purpose. In [155], various machine learning methods are assessed to determine the best fit for burned area prediction. In [156], the study focuses on the use of unsupervised methods. In [157], spectral indices associated with burned areas are classified by a random forest algorithm. In [158], neural networks are used and the accuracy is evaluated. In [159], the authors use a random forest method to develop a model that assesses the relationship between the severity of the wildfire and the previous suite of environmental conditions.

Table 4. Results: post-wildfire and restoration.

\begin{tabular}{|c|c|c|c|c|c|}
\hline Ref. & Classification $^{1}$ & Detailed Application & $\begin{array}{l}\text { Machine Learning } \\
\text { Technique }\end{array}$ & $\begin{array}{l}\text { Case study } \\
\text { Location }\end{array}$ & $\begin{array}{c}\text { Model } \\
\text { Performance } \\
\text { Metrics Results }\end{array}$ \\
\hline [137] & $\mathrm{A}$ & $\begin{array}{l}\text { Incorporating predictors of local } \\
\text { meteorology, land-surface } \\
\text { characteristics, and } \\
\text { socio-economic variables to } \\
\text { predict monthly burned area }\end{array}$ & $\begin{array}{l}\text { Shapley Additive } \\
\text { Explanation }\end{array}$ & United States & \\
\hline [138] & A & $\begin{array}{l}\text { Examining how training data } \\
\text { properties affect fire severity } \\
\text { classification across forest, } \\
\text { woodland, and shrubland } \\
\text { communities }\end{array}$ & & Southern Australia & \\
\hline [139] & $\mathrm{A}$ & $\begin{array}{l}\text { Determining the main } \\
\text { environmental variables that } \\
\text { control fire severity in large fires }\end{array}$ & Random Forest & Iberian Peninsula & \\
\hline [140] & $\mathrm{A}$ & $\begin{array}{l}\text { Predicting the burned area of } \\
\text { forest fires and the occurrence } \\
\text { of large-scale forest fires }\end{array}$ & & Portugal & \\
\hline [141] & A & $\begin{array}{l}\text { Topological data analysis to } \\
\text { assess the final burned area }\end{array}$ & & United States & \\
\hline
\end{tabular}


Table 4. Cont.

\begin{tabular}{|c|c|c|c|c|c|}
\hline Ref. & Classification $^{1}$ & Detailed Application & $\begin{array}{l}\text { Machine Learning } \\
\text { Technique }\end{array}$ & $\begin{array}{l}\text { Case study } \\
\text { Location }\end{array}$ & $\begin{array}{c}\text { Model } \\
\text { Performance } \\
\text { Metrics Results }\end{array}$ \\
\hline [142] & $\mathrm{A}$ & Mapping wildland fires & $\begin{array}{c}\text { Support Vector } \\
\text { Regression and the } \\
\text { Adaptive } \\
\text { Neuro-Fuzzy } \\
\text { Inference System }\end{array}$ & $\begin{array}{l}\text { Jerash Province, } \\
\text { Jordan }\end{array}$ & \\
\hline [143] & $\mathrm{A}$ & $\begin{array}{l}\text { Determining relationships } \\
\text { existing between the triggering } \\
\text { of landslides and burnt areas } \\
\text { through processing of the } \\
\text { thematic maps of the burnt } \\
\text { areas and landslide } \\
\text { susceptibility assessment }\end{array}$ & & & \\
\hline [136] & A & $\begin{array}{l}\text { Developing models for } \\
\text { automatically mapping burned } \\
\text { areas from unitemporal } \\
\text { multispectral imagery }\end{array}$ & & & \\
\hline [144] & $\mathrm{A}$ & $\begin{array}{l}\text { Assessing burn severity across } \\
\text { the burn scars and testing the } \\
\text { effectiveness of several remote } \\
\text { sensing methods for generating } \\
\text { accurate map products }\end{array}$ & $\begin{array}{c}\text { Random Forest } \\
\text { and Support Vector } \\
\text { Machine }\end{array}$ & Interior of Alaska & \\
\hline [145] & A & $\begin{array}{l}\text { Mapping of burned areas using } \\
\text { microwave data }\end{array}$ & Random Forests & & \\
\hline [146] & A & $\begin{array}{l}\text { Identifying burned areas and } \\
\text { estimating the fire history }\end{array}$ & Random Forest & $\begin{array}{l}\text { North Carolina, } \\
\text { United States }\end{array}$ & \\
\hline [147] & A & $\begin{array}{l}\text { Using automatic algorithm } \\
\text { approach to map burned areas } \\
\text { from remote sensing }\end{array}$ & & & \\
\hline [148] & A & $\begin{array}{l}\text { Examining the use of sUAS } \\
\text { imagery to train and validate } \\
\text { burn severity and extent } \\
\text { mapping of large wildland fires } \\
\text { from various satellite images }\end{array}$ & & & \\
\hline [149] & A & $\begin{array}{l}\text { Calculating tree mortality } \\
\text { through the comparison of } \\
\text { hyperspatial post-fire canopy } \\
\text { cover and pre-fire canopy cover }\end{array}$ & $\begin{array}{c}\text { Mask } \\
\text { Region-Based } \\
\text { Convolutional } \\
\text { Neural Network }\end{array}$ & & \\
\hline$[150]$ & A & $\begin{array}{l}\text { Determining trees and burned } \\
\text { pixels in a post-fire forest }\end{array}$ & $\begin{array}{c}\text { Mask } \\
\text { Region-Based } \\
\text { Convolutional } \\
\text { Neural Network } \\
\text { and Support Vector } \\
\text { Machine }\end{array}$ & & \\
\hline [151] & A & $\begin{array}{l}\text { Analysing bi-temporal (pre- and } \\
\text { post-fire) reflectance contrast of } \\
\text { burn-sensitive spectral bands }\end{array}$ & $\begin{array}{c}\text { Classification } \\
\text { Regression Tree, } \\
\text { Random Forest, } \\
\text { and Support Vector } \\
\text { Machine }\end{array}$ & & \\
\hline
\end{tabular}


Table 4. Cont.

\begin{tabular}{|c|c|c|c|c|c|}
\hline Ref. & Classification $^{1}$ & Detailed Application & $\begin{array}{l}\text { Machine Learning } \\
\text { Technique }\end{array}$ & $\begin{array}{l}\text { Case study } \\
\text { Location }\end{array}$ & $\begin{array}{c}\text { Model } \\
\text { Performance } \\
\text { Metrics Results }\end{array}$ \\
\hline [155] & $\mathrm{A}$ & $\begin{array}{l}\text { Mapping burned and unburned } \\
\text { areas, differentiating fire } \\
\text { occurrence dates, and } \\
\text { distinguishing between old and } \\
\text { more recent fires }\end{array}$ & $\begin{array}{c}\text { K-Nearest } \\
\text { Neighbours } \\
\text { Algorithm (K-NN), } \\
\text { Support Vector } \\
\text { Machine (SVM) } \\
\text { And Random } \\
\text { Forest }\end{array}$ & $\begin{array}{l}\text { Mediterranean } \\
\text { area }\end{array}$ & \\
\hline [156] & A & $\begin{array}{l}\text { Investigates the use capability } \\
\text { of the free synthetic aperture } \\
\text { radar data for burned area } \\
\text { mapping }\end{array}$ & & Portugal and Italy & \\
\hline [157] & $\mathrm{A}$ & $\begin{array}{l}\text { Test the applicability of a } \\
\text { normalized difference spectral } \\
\text { index with the shortwave } \\
\text { infrared and blue spectral bands } \\
\text { in accurately mapping burned } \\
\text { areas }\end{array}$ & Random Forest & & \\
\hline [158] & A & $\begin{array}{l}\text { Estimating burned areas in } \\
\text { forest fires }\end{array}$ & $\begin{array}{l}\text { Artificial Neural } \\
\text { Network }\end{array}$ & & \\
\hline [159] & A & $\begin{array}{l}\text { Examine the fine-scale } \\
\text { association between burn } \\
\text { severity and a suite of } \\
\text { environmental drivers }\end{array}$ & Random Forest & $\begin{array}{l}\text { California, United } \\
\text { States }\end{array}$ & $\begin{array}{l}\text { Accuracy of } 79.0 \% \\
\text { in classifying } \\
\text { categories of burn } \\
\text { severity }\end{array}$ \\
\hline [160] & $\mathrm{B}$ & $\begin{array}{l}\text { Evaluate public health impacts } \\
\text { of wildfire smoke }\end{array}$ & $\begin{array}{c}\text { Ordinary } \\
\text { Multi-Linear } \\
\text { Regression } \\
\text { Method, } \\
\text { Generalized } \\
\text { Boosting Method, } \\
\text { and Random } \\
\text { Forest }\end{array}$ & United States & \\
\hline [161] & $\mathrm{B}$ & $\begin{array}{l}\text { Prediction models for } \\
\text { ground-level ozone during } \\
\text { wildfires, evaluating the } \\
\text { predictive accuracy }\end{array}$ & & $\begin{array}{l}\text { California, United } \\
\text { States }\end{array}$ & \\
\hline [162] & B & $\begin{array}{l}\text { Explored different combinations } \\
\text { of biophysical and social factors } \\
\text { to characterize wildfire-affected } \\
\text { areas }\end{array}$ & Classification Trees & Portugal & \\
\hline [163] & $\mathrm{C}$ & $\begin{array}{l}\text { The carbon flux of the } \\
\text { woodland was monitored to } \\
\text { simulate daily net ecosystem } \\
\text { production, ecosystem } \\
\text { respiration, and gross primary } \\
\text { production }\end{array}$ & & & \\
\hline [164] & $\mathrm{C}$ & $\begin{array}{l}\text { Calculating emissions } \\
\text { associated with forest fires in } \\
\text { Mexico, based on different } \\
\text { satellite observation products }\end{array}$ & Random Forest & $\begin{array}{l}\text { Mexico and United } \\
\text { States }\end{array}$ & \\
\hline
\end{tabular}


Table 4. Cont.

\begin{tabular}{|c|c|c|c|c|c|}
\hline Ref. & Classification $^{1}$ & Detailed Application & $\begin{array}{l}\text { Machine Learning } \\
\text { Technique }\end{array}$ & $\begin{array}{l}\text { Case study } \\
\text { Location }\end{array}$ & $\begin{array}{c}\text { Model } \\
\text { Performance } \\
\text { Metrics Results }\end{array}$ \\
\hline [135] & $\mathrm{D}$ & $\begin{array}{l}\text { Proposes a unitemporal } \\
\text { simulation approach based on } \\
\text { the generation of synthetic } \\
\text { spectral databases from linear } \\
\text { spectral mixing to classify } \\
\text { wildfire severity }\end{array}$ & Random Forest & Spain & $\begin{array}{c}\text { Accuracy between } \\
90.0-95.0 \%\end{array}$ \\
\hline [165] & $\mathrm{D}$ & $\begin{array}{l}\text { Prediction of post-fire tree } \\
\text { mortality }\end{array}$ & Random Forest & & $\begin{array}{l}\text { Reduced the bias } \\
\text { in comparison } \\
\text { with logistic } \\
\text { regression method }\end{array}$ \\
\hline [166] & $\mathrm{D}$ & $\begin{array}{l}\text { Focus on the post-fire debris } \\
\text { flow hazards analysis }\end{array}$ & $\begin{array}{l}\text { Decision Tree } \\
\text { Algorithm }\end{array}$ & & $\begin{array}{c}\text { Sensitivity of } 81.0 \% \\
\text { and specificity of } \\
78.0 \%\end{array}$ \\
\hline [167] & $\mathrm{D}$ & $\begin{array}{c}\text { Map forest disturbance after a } \\
\text { wildfire }\end{array}$ & $\begin{array}{l}\text { Multiple Linear } \\
\text { Regression, } \\
\text { Support Vector } \\
\text { Machine, and } \\
\text { Random Forest }\end{array}$ & $\begin{array}{l}\text { Northeastern } \\
\text { China }\end{array}$ & \\
\hline
\end{tabular}

Identify temporal trends in post-fire regeneration and influences of climate on

[168] D post-fire regeneration, with focus on post-fire establishment, initial post-fire density and radial growth

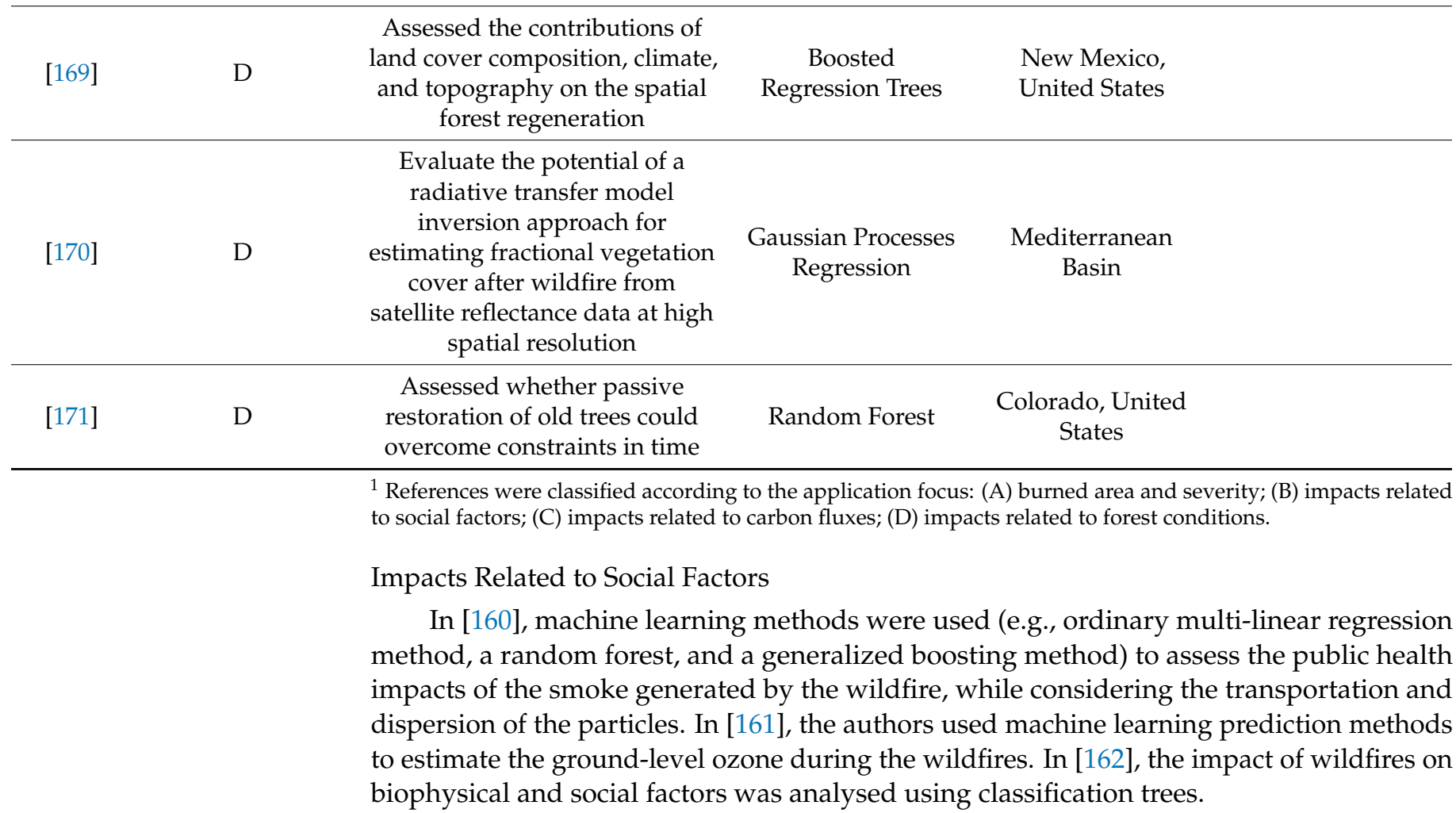




\section{Impacts Related to Carbon Fluxes}

In [163], the carbon fluxes (carbon source and carbon sink) related to carbon sequestration were assessed using a machine learning regression technique, by analysing pre- and post-wildfire data, to contribute to post-fire ecology-related decision-making. In [164], the authors develop an approach based on a random forest regression model for estimating emissions associated with wildfires, using satellite observations.

\section{Impacts Related to Forest Conditions}

In [135], the authors used random forest to develop four severity category classifications, where the model can detect the impacts of the wildfire on forests by classifying them as unburned, partial canopy unburned, canopy scorched, or canopy consumed. In [165], the authors predict tree mortality by employing a random forest model, and also evaluate the bias related to the model, in comparison with logistic regression.

In [166], a decision tree model is developed for the inductive inference of categorical data characterization, aiming at the post-fire analysis of debris hazards. In [167], stepwise multiple linear regression, support vector machine, and random forest were employed to analyse the statistical relationships between the recovery of the post-fire vegetation and the factors influencing it. In [168], the authors used a combination of dendro-ecological methods and machine learning to focus on the post-fire forest generation, taking into consideration factors related to climate, topographic variation, and pre-fire structure. In [169], a boosted regression trees method is used to assess the contribution of factors such as cover composition, topography, and climate on the vegetation.

In [170], the authors study the impact of wildfires on top-of-canopy spectral reflectance, by means the of Gaussian processes regression method. The authors in [171] focused on a combination of GIS and random forest methods to assess the passive restoration of old trees over time.

Table 4 presents in more details the classification of the mentioned articles considering the post-wildfire and restoration applications.

\subsection{Machine-Learning-Based Model Features and Feature Selection Sensitivity Analysis}

The use of high-dimensional data to train a model is a challenge in the application of machine learning. To address this, feature selection is used to remove irrelevant data or data with a lower impact on the model performance. The papers that explicitly presented a feature selection process aimed to select the best predictors by employing recursive feature elimination. In this framework, the size of the initial set of variables is recursively reduced, and an important related factor is attributed to each variable (as described in [88]), providing insights into which physical process associated with the variable with the biggest impact on the accuracy of the model. In order to contribute to the understanding the complex interrelationships between anthropogenic controls, wildfires, and the environment, while increasing the efficiency of decision-making support by removing unnecessary data, a feature selection process is developed in $[3,61,62,64,99,110,117]$. This is influential in reducing the computational time, improving the accuracy of the learning process, and facilitating the interpretation of the results [172].

\subsection{Identified Research Trends and Challenges}

This review concluded that there is an obvious potential for adopting machine learning methods for forecasting and classification, in different stages of the wildfire management cycle.

First, we list the trends identified. The category of pre-fire prevention and preparedness was the one most addressed by the reviewed papers. Most machine learning applications in the pre-fire stage (Table 2) focused on the two broad categories of risk assessment and ignition prediction of wildfires $(78.4 \%)$, followed by the use of machine learning for wildfire fuel modelling $(13.7 \%)$. The remaining applications for this stage accounted for $7.9 \%$ of the studies. 
Most research focusing on the management of active wildfires reported the use of machine learning for wildfire detection (61.9\%), followed by its use for wildfire spread prediction (23.8\%), and wildfire suppression (14.3\%).

In the post-wildfire stage, e.g., restoration and adaptation activities, machine learning approaches were mostly used for the assessment of burned areas severity (61.8\%), followed by applications for the assessment of impacts related to post-fire forest conditions (23.5\%), with the remaining applications accounting for $14.7 \%$ of the studies. A summary of research trends from the literature review includes:

- The increase in the use of ensemble modelling, which can reduce the inaccuracy and the computational time of the models by combining different models and machine learning techniques.

- The shifting focus from obtaining detailed physical interpretations to obtaining faster results based on input and output variables, especially at the stages in which the computational time of the modelling process is of critical importance, such as when dealing with active wildfires.

- The increasing concern with reducing the subjective bias associated with expertopinion-based methods, as well as with incorporating uncertainty analysis at the modelling or sub-modelling stages.

- The use in many papers of the association of remote sensing imageries, machine learning, and geospatial analysis to predict or classify variables of interest, in order to identify areas that are prone to wildfire occurrence.

- The exploration of classification and prediction using the main machine learning methods of random forest, support vector machine, and neural networks.

- The use, in many documents, of the association of multiple methods to obtain a more accurate set of candidate models for the same area, to provide a more refined sampling and to choose a final model, indicating that the researchers are exploring an integrated approach to benefit from the capabilities of different methods to assess the complex problem of wildfire modelling.

A variety of challenges still remain, associated with the use of machine learning methods to support wildfire management. Some of them are:

- $\quad$ The need for constant improvement, mainly to obtain faster models while enhancing the interpretability of the results. This is still a critical factor in machine-learning-based models.

- Overall, the studies did not present the computational time associated with the modelling process, which is necessary to evaluate the applicability of the models in such an important field as disaster management.

- $\quad$ Few studies developed feature selection to increase the efficiency of decision support.

- Lack of information about simulation platforms, precluding the comparison of the computational efficiency of different tools.

- $\quad$ Lack of experience of using global models that can be applied to different regions and with different datasets and thus of assessing the potential for generalization of the models via a parametric study of bias-variance trade-offs.

- Few studies are available that focus on the multifunctionality of forested landscape management planning, i.e., on integrating wildfire protection concerns in contexts characterized by demands for multiple ecosystem services.

- There is almost no analysis on the bare minimum amount of data needed for a useful model, especially for the active wildfire stage.

- Despite the fact that some methods deal with uncertainty, when models encompass forecasting the uncertainty may be substantial, thus impacting the results (as in the case of models that consider weather features) associated with management prescriptions.

- There are still few datasets for extensive wildfires, and most of the models are developed using smaller wildfire events for training, which may not correctly reflect the context of extreme wildfire events. 
- The acquisition of landscape dynamics data usually requires migrating data between datasets to quantify spatial patterns and changes through space and time. More developments are still required in this field.

- $\quad$ There may be issues regarding model overfitting that still need to be addressed.

- There is a need to bridge the gaps between monitorization and learning, and the decision-making process.

- There is a need for broader models that can help integrate the different wildfire management stages.

\section{Conclusions}

This review contributed to the characterization of the state of the art on the application of machine learning techniques to wildfire management decision support. It provided a summary of 135 recent papers (published between 2019 and 2022) that used machine learning approaches to address wildfire management issues. It also provided a classification of these approaches according to the area of application, machine learning method, case study location, and performance metrics.

In the case of the pre-fire prevention and preparedness stage, the main applications identified were segmented into the following categories: wildfire fuel modelling, risk assessment and ignition prediction of wildfires, support to dispatch, landscape planning and prevention measures for severity mitigation, and inventory data. In the case of the stage of management of active wildfires, the main applications were segmented into wildfire detection, spread prediction, and suppression. Finally, in the case of the post-wildfire stage of restoration and adaptation activities, the main applications were segmented into the analysis of burned area and severity, impacts related to social factors, impacts related to carbon fluxes, and impacts related to forest conditions.

The literature highlights that the main machine learning methods used for wildfire management decision support are random forest, support vector machine, and neural networks, and that both classification and prediction are explored. Overall, there is a trend towards the use of ensemble modelling to improve the accuracy of the models, as well as towards the integration of remote sensing imageries, machine learning, and geospatial analysis to identify areas that are prone to wildfire occurrence. The literature highlights further the potential for the association of multiple modelling methods to obtain a more accurate set of candidate models for the same area, to provide a more refined sampling in order to choose a final model.

There are still many challenges associated with a wider use of machine learning methods. Constant improvement is needed, mainly to obtain faster models while improving the interpretability of the results. There must also be a focus on the study of the computational time required for the modelling process, as well as a trade-off analysis between the computational time, platform simulation, feature selection, and management prescriptions related to the results obtained by the models.

Substantial steps are being taken to promote the use of machine learning methods within the framework of wildfire modelling applications. This review concluded that there is an obvious potential for adopting machine learning methods for forecasting and classification and for enhancing management decision support. It listed further challenges related to the fulfillment of this potential.

Author Contributions: Conceptualization, K.B. and J.G.B.; methodology, K.B. and J.G.B.; formal analysis, K.B. and J.G.B.; data curation, K.B.; writing-original draft preparation, K.B. and J.G.B.; writing-review and editing, K.B. and J.G.B.; project administration, J.G.B.; funding acquisition, J.G.B. All authors have read and agreed to the published version of the manuscript. 
Funding: This research was funded by the European Union's Horizon 2020 Research and Innovation Programme (grant agreement number 101037419_FIRE-RES project), by the Portuguese Science Foundation (FCT), Portugal, through the Forest Research Center Project UIDB/00239/2020, the BIOECOSYS project (grant number LISBOA-01-0145-FEDER-030391, PTDC/ASP-SIL/30391/2017) and the MODFIRE project (grant number PCIF/MOS/0217/2017).

Conflicts of Interest: The authors declare no conflict of interest.

\section{References}

1. Malik, A.; Rao, M.R.; Puppala, N.; Koouri, P.; Thota, V.A.K.; Liu, Q.; Chiao, S.; Gao, J. Data-Driven Wildfire Risk Prediction in Northern California. Atmosphere 2021, 12, 109. [CrossRef]

2. Carrasco, J.; Acuna, M.; Miranda, A.; Alfaro, G.; Pais, C.; Weintraub, A. Exploring the multidimensional effects of human activity and land cover on fire occurrence for territorial planning. J. Environ. Manag. 2021, 297, 113428. [CrossRef] [PubMed]

3. Pais, C.; Miranda, A.; Carrasco, J.; Shen, Z.-J.M. Deep fire topology: Understanding the role of landscape spatial patterns in wildfire occurrence using artificial intelligence. Environ. Model. Softw. 2021, 143, 105122. [CrossRef]

4. Banerjee, P. Maximum entropy-based forest fire likelihood mapping: Analysing the trends, distribution, and drivers of forest fires in Sikkim Himalaya. Scand. J. For. Res. 2021, 36, 275-288. [CrossRef]

5. $\quad$ Rego, F.; Rigolot, E.; Fernandes, P.; Montiel, C.; Silva, J.S. Towards Integrated Fire Management. European Forest Institute Policy Brief 4; EFI: Joensuu, Finland, 2010. Available online: http//www.efi.int/files/attachments/publications/efi_policy_brief_4_en.pdf (accessed on 25 October 2021).

6. Rego, F.M.C.C.; Rodríguez, J.M.M.; Calzada, V.R.V.; Xanthopoulos, G. Forest Fires: Sparking Firesmart Policies in the EU; European Comission: Brussel, Belgium, 2019.

7. Botequim, B.; Fernandes, P.M.; Borges, J.G.; González-Ferreiro, E.; Guerra-Hernández, J. Improving silvicultural practices for Mediterranean forests through fire behaviour modelling using LiDAR-derived canopy fuel characteristics. Int. J. Wildl. Fire 2019, 28, 823-839. [CrossRef]

8. Jain, P.; Coogan, S.C.P.; Subramanian, S.G.; Crowley, M.; Taylor, S.; Flannigan, M.D. A review of machine learning applications in wildfire science and management. Environ. Rev. 2020, 28, 478-505. [CrossRef]

9. Botequim, B.; Fernandes, P.M.; Garcia-Gonzalo, J.; Silva, A.; Borges, J.G. Coupling fire behaviour modelling and stand characteristics to assess and mitigate fire hazard in a maritime pine landscape in Portugal. Eur. J. For. Res. 2017, 136, 527-542. [CrossRef]

10. Marques, S.; Marto, M.; Bushenkov, V.; McDill, M.; Borges, J. Addressing wildfire risk in forest management planning with multiple criteria decision making methods. Sustainability 2017, 9, 298. [CrossRef]

11. EU Horizon 2020 Work Programme 2018-2020 Cross-Cutting Activities; Publications Office of the European Union: Luxemburg, 2020; pp. 70-73.

12. Pacheco, A.P.; Claro, J.; Fernandes, P.; de Neufville, R.; Oliveira, T.M.; Borges, J.G.; Rodrigues, J.C. Cohesive fire management within an uncertain environment: A review of risk handling and decision support systems. For. Ecol. Manag. 2015, $347,1-17$. [CrossRef]

13. Moreira, F.; Ascoli, D.; Safford, H.; A Adams, M.; Moreno, J.M.; Pereira, J.C.; Catry, F.X.; Armesto, J.; Bond, W.J.; E González, M.; et al. Wildfire management in Mediterranean-type regions: Paradigm change needed. Environ. Res. Lett. 2019, $15,011001$. [CrossRef]

14. Fernandes, P.; Botelho, H. Analysis of the prescribed burning practice in the pine forest of northwestern Portugal. J. Environ. Manag. 2004, 70, 15-26. [CrossRef] [PubMed]

15. Rigolot, E. Predicting postfire mortality of Pinus halepensis Mill. and Pinus pinea L. Plant Ecol. 2004, 171, 139-151. [CrossRef]

16. Marques, S.; Borges, J.G.; Garcia-Gonzalo, J.; Moreira, F.; Carreiras, J.; Oliveira, M.; Cantarinha, A.; Botequim, B.; Pereira, J.M.C. Characterization of wildfires in Portugal. Forstwiss. Cent. 2011, 130, 775-784. [CrossRef]

17. Marques, S.; Garcia-Gonzalo, J.; Botequim, B.; Ricardo, A.; Borges, J.G.; Tomé, M.; Oliveira, M. Assessing wildfire occurrence probability in Pinus pinaster Ait. stands in Portugal. For. Syst. 2012, 21, 111. [CrossRef]

18. Botequim, B.; Arias-Rodil, M.; Garcia-Gonzalo, J.; Silva, A.; Marques, S.; Borges, J.G.; Oliveira, M.M.; Tomé, M. Modeling Post-Fire Mortality in Pure and Mixed Forest Stands in Portugal-A Forest Planning-Oriented Model. Sustainability 2017, 9, 390. [CrossRef]

19. Zhai, C.; Zhang, S.; Cao, Z.; Wang, X. Learning-based prediction of wildfire spread with real-time rate of spread measurement. Combust. Flame 2020, 215, 333-341. [CrossRef]

20. Acuna, M.A.; Palma, C.D.; Cui, W.; Martell, D.L.; Weintraub, A. Integrated spatial fire and forest management planning. Can. J. For. Res. 2010, 40, 2370-2383. [CrossRef]

21. Mell, W.E.; McDermott, R.J.; Forney, G.P. Wildland fire behavior modeling: Perspectives, new approaches and applications. In Proceedings of the 3rd Fire Behavior and Fuels Conference, Washington, DC, USA, 25-29 October 2010; pp. 25-29.

22. Duff, T.J.; Tolhurst, K.G. Operational wildfire suppression modelling: A review evaluating development, state of the art and future directions. Int. J. Wildland Fire 2015, 24, 735. [CrossRef]

23. Martell, D.L. A review of operational research studies in forest fire management. Can. J. For. Res. 1982, 12, 119-140. [CrossRef] 
24. Martell, D.L. A Review of Recent Forest and Wildland Fire Management Decision Support Systems Research. Curr. For. Rep. 2015, 1, 128-137. [CrossRef]

25. El Naqa, I.; Murphy, M.J. What is machine learning? In Machine Learning in Radiation Oncology; Springer: Berlin/Heidelberg, Germany, 2015; pp. 3-11.

26. Cunningham, P.; Cord, M.; Delany, S.J. Supervised learning. In Machine Learning Techniques for Multimedia; Springer: Berlin/Heidelberg, Germany, 2008; pp. 21-49.

27. Muhammad, I.; Yan, Z. Supervised machine learning approaches: A survey. ICTACT J. Soft Comput. 2015, 5, 946-952. [CrossRef]

28. Kelleher, J.D.; Mac Namee, B.; D'arcy, A. Fundamentals of Machine Learning for Predictive Data Analytics: Algorithms, Worked Examples, and Case Studies; MIT Press: Cambridge, MA, USA, 2020; ISBN 0262361108.

29. Rish, I. An Empirical Study of the Naive Bayes Classifier. In Proceedings of the IJCAI 2001 Workshop on Empirical Methods in Artificial Intelligence, New York, NY, USA, 2 November 2001; Volume 3, pp. 41-46.

30. Freund, Y.; Mason, L. The Alternating Decision Tree Learning Algorithm. In Proceedings of the ICML, San Francisco, CA, USA, 27-30 June 1999; Citeseer: University Park, PA, USA, 1999; Volume 99, pp. 124-133.

31. Lewis, R.J. An Introduction to Classification and Regression Tree (CART) Analysis. In Proceedings of the Annual Meeting of the Society for Academic Emergency Medicine, San Francisco, CA, USA, 22-25 May 2000; Citeseer: University Park, PA, USA, 2000; Volume 14

32. Breiman, L. Random forests. Mach. Learn. 2001, 45, 5-32. [CrossRef]

33. Miikkulainen, R.; Liang, J.; Meyerson, E.; Rawal, A.; Fink, D.; Francon, O.; Raju, B.; Shahrzad, H.; Navruzyan, A.; Duffy, N.; et al. Evolving Deep Neural Networks. In Artificial Intelligence in the Age of Neural Networks and Brain Computing; Elsevier BV: Amsterdam, The Netherlands, 2019; pp. 293-312.

34. Wang, J.M.; Fleet, D.J.; Hertzmann, A. Gaussian Process Dynamical Models. In Proceedings of the NIPS, Cambridge, MA, USA, 5-8 December 2005; Citeseer: University Park, PA, USA, 2005; Volume 18, p. 3.

35. Yegnanarayana, B. Artificial Neural Networks; PHI Learning Pvt. Ltd.: New Delhi, India, 2009; ISBN 8120312538.

36. Sivanandam, S.N.; Deepa, S.N. Genetic algorithms. In Introduction to Genetic Algorithms; Springer: Berlin/Heidelberg, Germany, 2008; pp. 15-37.

37. Mikolov, T.; Karafiát, M.; Burget, L.; Cernocký, J.; Khudanpur, S. Recurrent neural network based language model. In Proceedings of the Interspeech, Baltimore, MD, USA, 20 July 2010; Volume 2, pp. 1045-1048.

38. Jaynes, E. On the rationale of maximum-entropy methods. Proc. IEEE 1982, 70, 939-952.

39. Elith, J.; Leathwick, J.R.; Hastie, T. A working guide to boosted regression trees. J. Anim. Ecol. 2008, 77, 802-813. [CrossRef]

40. Peterson, L.E. K-nearest neighbor. Scholarpedia 2009, 4, 1883. [CrossRef]

41. Hearst, M.A.; Dumais, S.T.; Osuna, E.; Platt, J.; Scholkopf, B. Support vector machines. IEEE Intell. Syst. Their Appl. 1998, 13, 18-28. [CrossRef]

42. Likas, A.; Vlassis, N.; Verbeek, J.J. The global k-means clustering algorithm. Pattern Recognit. 2003, 36, 451-461. [CrossRef]

43. Kohonen, T. Self-Organizing Maps: Ophmization Approaches. In Artificial Neural Networks; Elsevier BV: Amsterdam, The Netherlands, 1991; pp. 981-990.

44. Rasmussen, C.E. The infinite Gaussian mixture model. In Proceedings of the NIPS, Cambridge, MA, USA, 29 November-4 December 1999; Citeseer: University Park, PA, USA, 1999; Volume 12, pp. 554-560.

45. Eddy, S.R. What is a hidden Markov model? Nat. Biotechnol. 2004, 22, 1315-1316. [CrossRef]

46. Fritzke, B. Some Competitive Learning Methods; Ruhr University: Bochum, Germany, 1997.

47. Belkina, A.C.; Ciccolella, C.O.; Anno, R.; Halpert, R.; Spidlen, J.; Snyder-Cappione, J.E. Automated optimized parameters for T-distributed stochastic neighbor embedding improve visualization and analysis of large datasets. Nat. Commun. 2019, 10, 1-12. [CrossRef]

48. Miranda, A.; Carrasco, J.; González, M.; Pais, C.; Lara, A.; Altamirano, A.; Weintraub, A.; Syphard, A.D. Evidence-based mapping of the wildland-urban interface to better identify human communities threatened by wildfires. Environ. Res. Lett. 2020, 15, 094069. [CrossRef]

49. Naderpour, M.; Rizeei, H.M.; Khakzad, N.; Pradhan, B. Forest fire induced Natech risk assessment: A survey of geospatial technologies. Reliab. Eng. Syst. Saf. 2019, 191, 106558. [CrossRef]

50. Abid, F. A Survey of Machine Learning Algorithms Based Forest Fires Prediction and Detection Systems. Fire Technol. 2021, 57, 559-590. [CrossRef]

51. Afonso, R.; Neves, A.; Damásio, C.V.; Pires, J.M.; Birra, F.; Santos, M.Y. Assessment of Interventions in Fuel Management Zones Using Remote Sensing. ISPRS Int. J. Geo-Inf. 2020, 9, 533. [CrossRef]

52. Linn, R.; Winterkamp, J.; Furman, J.; Williams, B.; Hiers, J.; Jonko, A.; O’Brien, J.; Yedinak, K.; Goodrick, S. Modeling Low Intensity Fires: Lessons Learned from 2012 RxCADRE. Atmosphere 2021, 12, 139. [CrossRef]

53. McCandless, T.C.; Kosovic, B.; Petzke, W. Enhancing wildfire spread modelling by building a gridded fuel moisture content product with machine learning. Mach. Learn. Sci. Technol. 2020, 1, 035010. [CrossRef]

54. D’Este, M.; Elia, M.; Giannico, V.; Spano, G.; Lafortezza, R.; Sanesi, G. Machine Learning Techniques for Fine Dead Fuel Load Estimation Using Multi-Source Remote Sensing Data. Remote Sens. 2021, 13, 1658. [CrossRef]

55. Lee, H.; Won, M.; Yoon, S.; Jang, K. Estimation of 10-Hour Fuel Moisture Content Using Meteorological Data: A Model Inter-Comparison Study. Forest 2020, 11, 982. [CrossRef] 
56. Chiang, C.-Y.; Barnes, C.; Angelov, P.; Jiang, R. Deep Learning-Based Automated Forest Health Diagnosis From Aerial Images. IEEE Access 2020, 8, 144064-144076. [CrossRef]

57. Thurn, N.A.; Wood, T.; Williams, M.R.; Sigman, M.E. Classification of ground-truth fire debris samples using artificial neural networks. Forensic Chem. 2021, 23, 100313. [CrossRef]

58. Sigman, M.E.; Williams, M.R.; Thurn, N.; Wood, T. Validation of Ground Truth Fire Debris Classification by Supervised Machine Learning. Forensic Chem. 2021, 100358. [CrossRef]

59. Lauer, C.J.; A Montgomery, C.; Dietterich, T.G. Managing Fragmented Fire-Threatened Landscapes with Spatial Externalities. For Sci. 2019, 66, 443-456. [CrossRef]

60. Phelps, N.; Woolford, D.G. Comparing calibrated statistical and machine learning methods for wildland fire occurrence prediction: A case study of human-caused fires in Lac La Biche, Alberta, Canada. Int. J. Wildland Fire 2021. [CrossRef]

61. Shirazi, Z.; Wang, L.; Bondur, V.G. Modeling Conditions Appropriate for Wildfire in South East China-A Machine Learning Approach. Front. Earth Sci. 2021, 9. [CrossRef]

62. Campos-Vargas, C.; Vargas-Sanabria, D. Assessing the probability of wildfire occurrences in a neotropical dry forest. Écoscience 2021, 28, 159-169. [CrossRef]

63. Eskandari, S.; Pourghasemi, H.R.; Tiefenbacher, J.P. Relations of land cover, topography, and climate to fire occurrence in natural regions of Iran: Applying new data mining techniques for modeling and mapping fire danger. For. Ecol. Manag. 2020, 473, 118338. [CrossRef]

64. Xu, Z.; Liu, D.; Yan, L. Temperature-based fire frequency analysis using machine learning: A case of Changsha, China. Clim. Risk Manag. 2021, 31, 100276. [CrossRef]

65. Janiec, P.; Gadal, S. A Comparison of Two Machine Learning Classification Methods for Remote Sensing Predictive Modeling of the Forest Fire in the North-Eastern Siberia. Remote Sens. 2020, 12, 4157. [CrossRef]

66. Ghorbanzadeh, O.; Blaschke, T.; Gholamnia, K.; Aryal, J. Forest Fire Susceptibility and Risk Mapping Using Social/Infrastructural Vulnerability and Environmental Variables. Fire 2019, 2, 50. [CrossRef]

67. Silva, I.; Valle, M.; Barros, L.; Meyer, J. A wildfire warning system applied to the state of Acre in the Brazilian Amazon. Appl. Soft Comput. 2020, 89, 106075. [CrossRef]

68. Choi, M.-Y.; Jun, S. Fire Risk Assessment Models Using Statistical Machine Learning and Optimized Risk Indexing. Appl. Sci. 2020, 10, 4199. [CrossRef]

69. Milanović, S.; Marković, N.; Pamučar, D.; Gigović, L.; Kostić, P.; Milanović, S. Forest Fire Probability Mapping in Eastern Serbia: Logistic Regression versus Random Forest Method. Forests 2020, 12, 5. [CrossRef]

70. Kang, Y.; Jang, E.; Im, J.; Kwon, C.; Kim, S. Developing a New Hourly Forest Fire Risk Index Based on Catboost in South Korea. Appl. Sci. 2020, 10, 8213. [CrossRef]

71. Kim, S.J.; Lim, C.-H.; Kim, G.S.; Lee, J.; Geiger, T.; Rahmati, O.; Son, Y.; Lee, W.-K. Multi-Temporal Analysis of Forest Fire Probability Using Socio-Economic and Environmental Variables. Remote Sens. 2019, 11, 86. [CrossRef]

72. Bjånes, A.; De La Fuente, R.; Mena, P. A deep learning ensemble model for wildfire susceptibility mapping. Ecol. Inform. 2021, 65, 101397. [CrossRef]

73. Tonini, M.; D’Andrea, M.; Biondi, G.; Degli Esposti, S.; Trucchia, A.; Fiorucci, P. A Machine Learning-Based Approach for Wildfire Susceptibility Mapping. The Case Study of the Liguria Region in Italy. Geosciences 2020, 10, 105. [CrossRef]

74. Tehrany, M.S.; Jones, S.; Shabani, F.; Martínez-Álvarez, F.; Bui, D.T. A novel ensemble modeling approach for the spatial prediction of tropical forest fire susceptibility using LogitBoost machine learning classifier and multi-source geospatial data. Arch. Meteorol. Geophys. Bioclimatol. Ser. B 2019, 137, 637-653. [CrossRef]

75. Achu, A.; Thomas, J.; Aju, C.; Gopinath, G.; Kumar, S.; Reghunath, R. Machine-learning modelling of fire susceptibility in a forest-agriculture mosaic landscape of southern India. Ecol. Inform. 2021, 64, 101348. [CrossRef]

76. Gigović, L.; Pourghasemi, H.R.; Drobnjak, S.; Bai, S. Testing a New Ensemble Model Based on SVM and Random Forest in Forest Fire Susceptibility Assessment and Its Mapping in Serbia's Tara National Park. Forests 2019, 10, 408. [CrossRef]

77. Sánchez, M.B.; Tonini, M.; Mapelli, A.; Fiorucci, P. Spatial Assessment of Wildfires Susceptibility in Santa Cruz (Bolivia) Using Random Forest. Geosciences 2021, 11, 224. [CrossRef]

78. PPham, B.T.; Jaafari, A.; Avand, M.; Al-Ansari, N.; Dinh Du, T.; Yen, H.P.H.; Phong, T.V.; Nguyen, D.H.; Le, H.V.; Mafi-Gholami, D.; et al. Performance Evaluation of Machine Learning Methods for Forest Fire Modeling and Prediction. Symmetry 2020, $12,1022$. [CrossRef]

79. Eskandari, S.; Amiri, M.; Sãdhasivam, N.; Pourghasemi, H.R. Comparison of new individual and hybrid machine learning algorithms for modeling and mapping fire hazard: A supplementary analysis of fire hazard in different counties of Golestan Province in Iran. Nat. Hazards 2020, 104, 305-327. [CrossRef]

80. Yousefi, S.; Pourghasemi, H.R.; Emami, S.N.; Pouyan, S.; Eskandari, S.; Tiefenbacher, J.P. A machine learning framework for multi-hazards modeling and mapping in a mountainous area. Sci. Rep. 2020, 10, 1-14. [CrossRef] [PubMed]

81. Michael, Y.; Helman, D.; Glickman, O.; Gabay, D.; Brenner, S.; Lensky, I.M. Forecasting fire risk with machine learning and dynamic information derived from satellite vegetation index time-series. Sci. Total Environ. 2021, 764, 142844. [CrossRef] [PubMed]

82. Mohajane, M.; Costache, R.; Karimi, F.; Pham, Q.B.; Essahlaoui, A.; Nguyen, H.; Laneve, G.; Oudija, F. Application of remote sensing and machine learning algorithms for forest fire mapping in a Mediterranean area. Ecol. Indic. 2021, 129, 107869. [CrossRef] 
83. Tuyen, T.T.; Jaafari, A.; Yen, H.P.H.; Nguyen-Thoi, T.; Van Phong, T.; Nguyen, H.D.; Van Le, H.; Phuong, T.T.M.; Nguyen, S.H.; Prakash, I.; et al. Mapping forest fire susceptibility using spatially explicit ensemble models based on the locally weighted learning algorithm. Ecol. Inform. 2021, 63, 101292. [CrossRef]

84. Tang, X.; Machimura, T.; Li, J.; Liu, W.; Hong, H. A novel optimized repeatedly random undersampling for selecting negative samples: A case study in an SVM-based forest fire susceptibility assessment. J. Environ. Manag. 2020, 271, 111014. [CrossRef]

85. Galizia, L.F.D.C.; Rodrigues, M. Modeling the Influence of Eucalypt Plantation on Wildfire Occurrence in the Brazilian Savanna Biome. Forests 2019, 10, 844. [CrossRef]

86. Elia, M.; D’Este, M.; Ascoli, D.; Giannico, V.; Spano, G.; Ganga, A.; Colangelo, G.; Lafortezza, R.; Sanesi, G. Estimating the probability of wildfire occurrence in Mediterranean landscapes using Artificial Neural Networks. Environ. Impact Assess. Rev. 2020, 85, 106474. [CrossRef]

87. Sayad, Y.O.; Mousannif, H.; Al Moatassime, H. Predictive modeling of wildfires: A new dataset and machine learning approach Fire Saf. J. 2019, 104, 130-146. [CrossRef]

88. Coughlan, R.; Di Giuseppe, F.; Vitolo, C.; Barnard, C.; Lopez, P.; Drusch, M. Using machine learning to predict fire-ignition occurrences from lightning forecasts. Meteorol. Appl. 2021, 28, e1973. [CrossRef]

89. Naderpour, M.; Rizeei, H.; Ramezani, F. Forest Fire Risk Prediction: A Spatial Deep Neural Network-Based Framework. Remote Sens. 2021, 13, 2513. [CrossRef]

90. Zhang, G.; Wang, M.; Liu, K. Forest Fire Susceptibility Modeling Using a Convolutional Neural Network for Yunnan Province of China. Int. J. Disaster Risk Sci. 2019, 10, 386-403. [CrossRef]

91. Pourghasemi, H.R.; Gayen, A.; Lasaponara, R.; Tiefenbacher, J.P. Application of learning vector quantization and different machine learning techniques to assessing forest fire influence factors and spatial modelling. Environ. Res. 2020, $184,109321$. [CrossRef]

92. Sharma, R.; Rani, S.; Memon, I. A smart approach for fire prediction under uncertain conditions using machine learning. Multimed. Tools Appl. 2020, 79, 28155-28168. [CrossRef]

93. Bui, D.T.; Hoang, N.-D.; Samui, P. Spatial pattern analysis and prediction of forest fire using new machine learning approach of Multivariate Adaptive Regression Splines and Differential Flower Pollination optimization: A case study at Lao Cai province (Viet Nam). J. Environ. Manag. 2019, 237, 476-487. [CrossRef]

94. Lattimer, B.; Hodges, J.; Lattimer, A. Using machine learning in physics-based simulation of fire. Fire Saf. J. 2020, $114,102991$. [CrossRef]

95. Barreto, J.; Armenteras, D. Open Data and Machine Learning to Model the Occurrence of Fire in the Ecoregion of "Llanos Colombo-Venezolanos". Remote Sens. 2020, 12, 3921. [CrossRef]

96. Hogland, J.; Dunn, C.J.; Johnston, J.D. 21st Century Planning Techniques for Creating Fire-Resilient Forests in the American West. Forests 2021, 12, 1084. [CrossRef]

97. Agarwal, P.; Tang, J.; Narayanan, A.N.L.; Zhuang, J. Big Data and Predictive Analytics in Fire Risk Using Weather Data. Risk Anal 2020, 40, 1438-1449. [CrossRef] [PubMed]

98. Yu, Y.; Mao, J.; Thornton, P.E.; Notaro, M.; Wullschleger, S.D.; Shi, X.; Hoffman, F.M.; Wang, Y. Quantifying the drivers and predictability of seasonal changes in African fire. Nat. Commun. 2020, 11, 1-8. [CrossRef] [PubMed]

99. Thompson, M.P.; Gannon, B.M.; Caggiano, M.D. Forest Roads and Operational Wildfire Response Planning. Forests 2021, 12, 110. [CrossRef]

100. Rodrigues, M.; Alcasena, F.; Vega-García, C. Modeling initial attack success of wildfire suppression in Catalonia, Spain. Sci. Total Environ. 2019, 666, 915-927. [CrossRef] [PubMed]

101. He, Q.; Jiang, Z.; Wang, M.; Liu, K. Landslide and Wildfire Susceptibility Assessment in Southeast Asia Using Ensemble Machine Learning Methods. Remote Sens. 2021, 13, 1572. [CrossRef]

102. Hanberry, B.B. Reclassifying the Wildland-Urban Interface Using Fire Occurrences for the United States. Land $2020,9,225$. [CrossRef]

103. Moustakas, A.; Davlias, O. Minimal effect of prescribed burning on fire spread rate and intensity in savanna ecosystems. Stoch Environ. Res. Risk Assess. 2021, 35, 1-12. [CrossRef]

104. Gholamnia, K.; Nachappa, T.G.; Ghorbanzadeh, O.; Blaschke, T. Comparisons of Diverse Machine Learning Approaches for Wildfire Susceptibility Mapping. Symmetry 2020, 12, 604. [CrossRef]

105. Sulova, A.; Arsanjani, J.J. Exploratory Analysis of Driving Force of Wildfires in Australia: An Application of Machine Learning within Google Earth Engine. Remote Sens. 2021, 13, 10. [CrossRef]

106. Ghorbanzadeh, O.; Kamran, K.V.; Blaschke, T.; Aryal, J.; Naboureh, A.; Einali, J.; Bian, J. Spatial Prediction of Wildfire Susceptibility Using Field Survey GPS Data and Machine Learning Approaches. Fire 2019, 2, 43. [CrossRef]

107. Pérez-Porras, F.-J.; Triviño-Tarradas, P.; Cima-Rodríguez, C.; Meroño-De-Larriva, J.-E.; García-Ferrer, A.; Mesas-Carrascosa, F.-J . Machine Learning Methods and Synthetic Data Generation to Predict Large Wildfires. Sensors 2021, 21, 3694. [CrossRef]

108. Yang, X.; Chen, R.; Zhang, F.; Zhang, L.; Fan, X.; Ye, Q.; Fu, L. Pixel-level automatic annotation for forest fire image. Eng. Appl. Artif. Intell. 2021, 104, 104353. [CrossRef]

109. Govil, K.; Welch, M.L.; Ball, J.T.; Pennypacker, C.R. Preliminary Results from a Wildfire Detection System Using Deep Learning on Remote Camera Images. Remote Sens. 2020, 12, 166. [CrossRef] 
110. Shi, J.; Wang, W.; Gao, Y.; Yu, N. Optimal Placement and Intelligent Smoke Detection Algorithm for Wildfire-Monitoring Cameras IEEE Access 2020, 8, 72326-72339. [CrossRef]

111. Munshi, A. Fire Detection Methods Based on Various Color Spaces and Gaussian Mixture Models. Adv. Sci. Technol. Res. J. 2021, 15, 197-214. [CrossRef]

112. Jang, E.; Kang, Y.; Im, J.; Lee, D.-W.; Yoon, J.; Kim, S.-K. Detection and Monitoring of Forest Fires Using Himawari-8 Geostationary Satellite Data in South Korea. Remote Sens. 2019, 11, 271. [CrossRef]

113. Nguyen, M.D.; Vu, H.N.; Pham, D.C.; Choi, B.; Ro, S. Multistage Real-time Fire Detection using Convolutional Neural Networks and Long Short-term Memory Networks. IEEE Access 2021, 9, 146667-146679. [CrossRef]

114. Liu, Z.; Zhang, K.; Wang, C.; Huang, S. Research on the identification method for the forest fire based on deep learning. Optik 2020, 223, 165491. [CrossRef]

115. Rossi, R.; Gelfusa, M.; Malizia, A.; Gaudio, P. Adaptive Quasi-Unsupervised Detection of Smoke Plume by LiDAR. Sensors 2020, 20, 6602. [CrossRef] [PubMed]

116. Saeed, F.; Paul, A.; Hong, W.H.; Seo, H. Machine learning based approach for multimedia surveillance during fire emergencies. Multimed. Tools Appl. 2020, 79, 16201-16217. [CrossRef]

117. Saeed, F.; Paul, A.; Karthigaikumar, P.; Nayyar, A. Convolutional neural network based early fire detection. Multimed. Tools Appl. 2019, 79, 9083-9099. [CrossRef]

118. Choi, M.; A Wi, J.; Kim, T.; Kim, Y.; Kim, C.-H. Learning Representation of Secondary Effects for Fire-Flake Animation. IEEE Access 2021, 9, 17620-17630. [CrossRef]

119. Amici, S.; Piscini, A. Exploring PRISMA Scene for Fire Detection: Case Study of 2019 Bushfires in Ben Halls Gap National Park, NSW, Australia. Remote Sens. 2021, 13, 1410. [CrossRef]

120. Ma, J.; Cheng, J.C.; Jiang, F.; Gan, V.J.; Wang, M.; Zhai, C. Real-time detection of wildfire risk caused by powerline vegetation faults using advanced machine learning techniques. Adv. Eng. Inform. 2020, 44, 101070. [CrossRef]

121. Gibson, R.; Danaher, T.; Hehir, W.; Collins, L. A remote sensing approach to mapping fire severity in south-eastern Australia using sentinel 2 and random forest. Remote Sens. Environ. 2020, 240, 111702. [CrossRef]

122. Imran; Iqbal, N.; Ahmad, S.; Kim, D. Towards Mountain Fire Safety Using Fire Spread Predictive Analytics and Mountain Fire Containment in IoT Environment. Sustainability 2021, 13, 2461. [CrossRef]

123. Farguell, A.; Mandel, J.; Haley, J.; Mallia, D.; Kochanski, A.; Hilburn, K. Machine Learning Estimation of Fire Arrival Time from Level-2 Active Fires Satellite Data. Remote Sens. 2021, 13, 2203. [CrossRef]

124. Hodges, J.L.; Lattimer, B.Y. Wildland Fire Spread Modeling Using Convolutional Neural Networks. Fire Technol. 2019, 55, 2115-2142. [CrossRef]

125. McCarthy, N.; Tohidi, A.; Aziz, Y.; Dennie, M.; Valero, M.; Hu, N. A Deep Learning Approach to Downscale Geostationary Satellite Imagery for Decision Support in High Impact Wildfires. Forests 2021, 12, 294. [CrossRef]

126. García-Llamas, P.; Suárez-Seoane, S.; Taboada, A.; Fernández-Manso, A.; Quintano, C.; Fernández-García, V.; FernándezGuisuraga, J.M.; Marcos, E.; Calvo, L. Environmental drivers of fire severity in extreme fire events that affect Mediterranean pine forest ecosystems. For. Ecol. Manag. 2019, 433, 24-32. [CrossRef]

127. Povak, N.A.; Kane, V.R.; Collins, B.M.; Lydersen, J.M.; Kane, J.T. Multi-scaled drivers of severity patterns vary across land ownerships for the 2013 Rim Fire, California. Landsc. Ecol. 2019, 35, 293-318. [CrossRef]

128. Coffield, S.R.; Graff, C.A.; Chen, Y.; Smyth, P.; Foufoula-Georgiou, E.; Randerson, J.T. Machine learning to predict final fire size at the time of ignition. Int. J. Wildland Fire 2019, 28, 861-873. [CrossRef] [PubMed]

129. Zohdi, T. A machine-learning framework for rapid adaptive digital-twin based fire-propagation simulation in complex environments. Comput. Methods Appl. Mech. Eng. 2020, 363, 112907. [CrossRef]

130. Zohdi, T. A digital twin framework for machine learning optimization of aerial fire fighting and pilot safety. Comput. Methods Appl. Mech. Eng. 2021, 373, 113446. [CrossRef]

131. Koklu, M.; Taspinar, Y.S. Determining the Extinguishing Status of Fuel Flames With Sound Wave by Machine Learning Methods. IEEE Access 2021, 9, 86207-86216. [CrossRef]

132. Taspinar, Y.S.; Koklu, M.; Altin, M. Classification of flame extinction based on acoustic oscillations using artificial intelligence methods. Case Stud. Therm. Eng. 2021, 28, 101561. [CrossRef]

133. Bhattarai, M.; Martinez-Ramon, M. A Deep Learning Framework for Detection of Targets in Thermal Images to Improve Firefighting. IEEE Access 2020, 8, 88308-88321. [CrossRef]

134. Farasin, A.; Colomba, L.; Garza, P. Double-Step U-Net: A Deep Learning-Based Approach for the Estimation of Wildfire Damage Severity through Sentinel-2 Satellite Data. Appl. Sci. 2020, 10, 4332. [CrossRef]

135. Montorio, R.; Pérez-Cabello, F.; Alves, D.B.; García-Martín, A. Unitemporal approach to fire severity mapping using multispectral synthetic databases and Random Forests. Remote Sens. Environ. 2020, 249, 249. [CrossRef]

136. Hu, X.; Ban, Y.; Nascetti, A. Uni-Temporal Multispectral Imagery for Burned Area Mapping with Deep Learning. Remote Sens. 2021, 13, 1509. [CrossRef]

137. Wang, S.S.; Qian, Y.; Leung, L.R.; Zhang, Y. Identifying Key Drivers of Wildfires in the Contiguous US Using Machine Learning and Game Theory Interpretation. Earth's Futur. 2021, 9, e2020EF001910. [CrossRef]

138. Collins, L.; McCarthy, G.; Mellor, A.; Newell, G.; Smith, L. Training data requirements for fire severity mapping using Landsat imagery and random forest. Remote Sens. Environ. 2020, 245, 111839. [CrossRef] 
139. García-Llamas, P.; Suárez-Seoane, S.; Fernández-Manso, A.; Quintano, C.; Calvo, L. Evaluation of fire severity in fire proneecosystems of Spain under two different environmental conditions. J. Environ. Manag. 2020, 271, 110706. [CrossRef]

140. Xie, Y.; Peng, M. Forest fire forecasting using ensemble learning approaches. Neural Comput. Appl. 2019, 31, 4541-4550. [CrossRef]

141. Bendick, R.; Hoylman, Z.H. Topological data analysis reveals parameters with prognostic skill for extreme wildfire size. Environ. Res. Lett. 2020, 15, 104039. [CrossRef]

142. Al-Fugara, A.; Mabdeh, A.; Ahmadlou, M.; Pourghasemi, H.; Al-Adamat, R.; Pradhan, B.; Al-Shabeeb, A. Wildland Fire Susceptibility Mapping Using Support Vector Regression and Adaptive Neuro-Fuzzy Inference System-Based Whale Optimization Algorithm and Simulated Annealing. ISPRS Int. J. Geo-Inf. 2021, 10, 382. [CrossRef]

143. Di Napoli, M.; Marsiglia, P.; Di Martire, D.; Ramondini, M.; Ullo, S.; Calcaterra, D. Landslide Susceptibility Assessment of Wildfire Burnt Areas through Earth-Observation Techniques and a Machine Learning-Based Approach. Remote Sens. 2020, 12, 2505. [CrossRef]

144. Smith, C.; Panda, S.; Bhatt, U.; Meyer, F.; Badola, A.; Hrobak, J. Assessing Wildfire Burn Severity and Its Relationship with Environmental Factors: A Case Study in Interior Alaska Boreal Forest. Remote Sens. 2021, 13, 1966. [CrossRef]

145. Carreiras, J.M.B.; Quegan, S.; Tansey, K.; Page, S. Sentinel-1 observation frequency significantly increases burnt area detectability in tropical SE Asia. Environ. Res. Lett. 2020, 15, 054008. [CrossRef]

146. Wall, W.A.; Hohmann, M.G.; Just, M.G.; Hoffmann, W.A. Characterizing past fire occurrence in longleaf pine ecosystems with the Mid-Infrared Burn Index and a Random Forest classifier. For. Ecol. Manag. 2021, 500, 119635. [CrossRef]

147. Stroppiana, D.; Bordogna, G.; Sali, M.; Boschetti, M.; Sona, G.; Brivio, P.A. A Fully Automatic, Interpretable and Adaptive Machine Learning Approach to Map Burned Area from Remote Sensing. ISPRS Int. J. Geo-Inf. 2021, 10, 546. [CrossRef]

148. Hamilton, D.; Levandovsky, E.; Hamilton, N. Mapping Burn Extent of Large Wildland Fires from Satellite Imagery Using Machine Learning Trained from Localized Hyperspatial Imagery. Remote Sens. 2020, 12, 4097. [CrossRef]

149. Hamilton, D.; Brothers, K.; Jones, S.; Colwell, J.; Winters, J. Wildland Fire Tree Mortality Mapping from Hyperspatial Imagery Using Machine Learning. Remote Sens. 2021, 13, 290. [CrossRef]

150. Hamilton, D.; Brothers, K.; McCall, C.; Gautier, B.; Shea, T. Mapping Forest Burn Extent from Hyperspatial Imagery Using Machine Learning. Remote Sens. 2021, 13, 3843. [CrossRef]

151. Bar, S.; Parida, B.R.; Pandey, A.C. Landsat-8 and Sentinel-2 based Forest fire burn area mapping using machine learning algorithms on GEE cloud platform over Uttarakhand, Western Himalaya. Remote Sens. Appl. Soc. Environ. 2020, 18, 100324. [CrossRef]

152. Belenguer-Plomer, M.A.; Tanase, M.A.; Fernandez-Carrillo, A.; Chuvieco, E. Burned area detection and mapping using Sentinel-1 backscatter coefficient and thermal anomalies. Remote Sens. Environ. 2019, 233, 233. [CrossRef]

153. Çömert, R.; Matci, D.K.; Avdan, U. Object based burned area mapping with random forest algorithm. Int. J. Eng. Geosci. 2019, 4, 78-87. [CrossRef]

154. Jiang, Y.; Zhou, L.; Raghavendra, A. Observed changes in fire patterns and possible drivers over Central Africa. Environ. Res. Lett. 2020, 15, 0940b8. [CrossRef]

155. Gelabert, P.J.; Montealegre, A.; Lamelas, M.; Domingo, D. Forest structural diversity characterization in Mediterranean landscapes affected by fires using Airborne Laser Scanning data. GIScience Remote Sens. 2020, 57, 497-509. [CrossRef]

156. De Luca, G.; Silva, J.M.; Modica, G. A workflow based on Sentinel-1 SAR data and open-source algorithms for unsupervised burned area detection in Mediterranean ecosystems. GIScience Remote Sens. 2021, 58, 516-541. [CrossRef]

157. Mpakairi, K.S.; Kadzunge, S.L.; Ndaimani, H. Testing the utility of the blue spectral region in burned area mapping: Insights from savanna wildfires. Remote Sens. Appl. Soc. Environ. 2020, 20, 100365. [CrossRef]

158. Calp, M.H.; Kose, U. Estimation of burned areas in forest fires using artificial neural networks. Ing. Solidar. 2020, 16, 1-22. [CrossRef]

159. Huang, Y.; Jin, Y.; Schwartz, M.W.; Thorne, J.H. Intensified burn severity in California's northern coastal mountains by drier climatic condition. Environ. Res. Lett. 2020, 15, 104033. [CrossRef]

160. Zou, Y.; O’Neill, S.M.; Larkin, N.K.; Alvarado, E.C.; Solomon, R.; Mass, C.; Liu, Y.; Odman, M.T.; Shen, H. Machine Learning-Based Integration of High-Resolution Wildfire Smoke Simulations and Observations for Regional Health Impact Assessment. Int. J. Environ. Res. Public Health 2019, 16, 2137. [CrossRef]

161. Watson, G.L.; Telesca, D.; Reid, C.; Pfister, G.G.; Jerrett, M. Machine learning models accurately predict ozone exposure during wildfire events. Environ. Pollut. 2019, 254, 112792. [CrossRef] [PubMed]

162. Oliveira, S.; Zêzere, J.L. Assessing the biophysical and social drivers of burned area distribution at the local scale. J. Environ. Manag. 2020, 264, 110449. [CrossRef] [PubMed]

163. Sun, Q.; Meyer, W.S.; Koerber, G.R.; Marschner, P. Rapid recovery of net ecosystem production in a semi-arid woodland after a wildfire. Agric. For. Meteorol. 2020, 291, 108099. [CrossRef]

164. Cruz-López, M.I.; Manzo-Delgado, L.D.L.; Aguirre-Gómez, R.; Chuvieco, E.; Equihua-Benítez, J.A. Spatial Distribution of Forest Fire Emissions: A Case Study in Three Mexican Ecoregions. Remote. Sens. 2019, 11, 1185. [CrossRef]

165. Shearman, T.M.; Varner, J.M.; Hood, S.M.; Cansler, C.A.; Hiers, J.K. Modelling post-fire tree mortality: Can random forest improve discrimination of imbalanced data? Ecol. Model. 2019, 414, 108855. [CrossRef]

166. Addison, P.; Oommen, T.; Sha, Q. Assessment of post-wildfire debris flow occurrence using classifier tree. Geomat. Nat. Hazards Risk 2019, 10, 505-518. [CrossRef] 
167. Qiu, J.; Wang, H.; Shen, W.; Zhang, Y.; Su, H.; Li, M. Quantifying Forest Fire and Post-Fire Vegetation Recovery in the Daxing'anling Area of Northeastern China Using Landsat Time-Series Data and Machine Learning. Remote. Sens. 2021, 13, 792. [CrossRef]

168. Guz, J.; Gill, N.S.; Kulakowski, D. Long-term empirical evidence shows post-disturbance climate controls post-fire regeneration. J. Ecol. 2021, 109, 4007-4024. [CrossRef]

169. Wang, J.; Zhang, X.; Rodman, K. Land cover composition, climate, and topography drive land surface phenology in a recently burned landscape: An application of machine learning in phenological modeling. Agric. For. Meteorol. 2021, 304-305, 108432. [CrossRef]

170. Fernández-Guisuraga, J.M.; Verrelst, J.; Calvo, L.; Suárez-Seoane, S. Hybrid inversion of radiative transfer models based on high spatial resolution satellite reflectance data improves fractional vegetation cover retrieval in heterogeneous ecological systems after fire. Remote Sens. Environ. 2021, 255, 112304. [CrossRef]

171. Baker, W.L. Restoration of forest resilience to fire from old trees is possible across a large Colorado dry-forest landscape by 2060, but only under the Paris $1.5^{\circ} \mathrm{C}$ goal. Glob. Chang. Biol. 2021, 27, 4074-4095. [CrossRef] [PubMed]

172. Cai, J.; Luo, J.; Wang, S.; Yang, S. Feature selection in machine learning: A new perspective. Neurocomputing 2018, 300, 70-79. [CrossRef] 